\title{
Developments in acoustics for studying wave-driven boundary layer flow and sediment dynamics over rippled sand-beds
}

3

4 Peter D. Thorne ${ }^{1 * *}$, David Hurther ${ }^{2}$, Richard D. Cooke ${ }^{1}$, Ivan Caceres ${ }^{3}$, Pierre. A. Barraud ${ }^{2 *}$ 5 and Agustín Sánchez-Arcilla ${ }^{3}$

6

71 National Oceanography Centre, Joseph Proudman Building, 6 Brownlow Street, Liverpool,

$8 \quad$ L3 5DA, United Kingdom.

9

102 Laboratory of Geophysical and Industrial Flows (LEGI), CNRS UMR 5519, University

11 Grenoble Alpes, France

12

133 Laboratori d'Enginyeria Marítima (LIM/UPC), Universitat Politècnica de Catalunya (UPC)-

14 Barcelonatech, Campus Nord, c./ Jordi Girona, 1-3. 08034 Barcelona, Spain.

15

$16 *$ Current affiliation TIMC, CNRS, University Grenoble Alpes, France

17

** Corresponding author

19 pdt@noc.ac.uk

20 National Oceanography Centre, Joseph Proudman Building, 6 Brownlow Street, Liverpool, 21 L3 5DA, United Kingdom.

Thorne, Peter D.; Hurther, David; Cooke, Richard D.; Caceres, Ivan; Barraud, Pierre A.; Sánchez-Arcilla, Agustín. 2018. Developments in acoustics for studying wave-driven boundary layer flow and sediment dynamics over rippled sand-beds. Continental Shelf Research, 166. 119-137. https://doi.org/10.1016/j.csr.2018.07.008 
3 The processes of sediment entrainment, transport, and deposition over bedforms are highly 4 dynamic and temporally and spatially variable. Obtaining measurements to understand these 5 processes has led to ongoing developments in instrumentation for studying near-bed sediment 6 dynamics, with the outputs applied to the development and assessment of sediment transport 7 modelling. In the present study results are reported from three acoustic systems deployed to 8 make observations of bedforms, bedload, suspended concentration and horizontal and vertical 9 velocity components. To evaluate the instruments a series of near-bed boundary layer 10 measurements were collected in a large scale flume facility over a rippled bed of medium sand 11 under regular waves. The observations were conducted as part of Joint Research Activities 12 within the EU funded Hydralab project. The suite of acoustic instruments consisted of a 13 Bedform And Suspended Sediment Imager, BASSI, a three dimensional acoustic ripple 14 profiler, 3D-ARP, and three Acoustic Concentration and Velocity Profilers, ACVP's. Here 15 results are reported from the deployment of the instruments, to illustrate the ongoing 16 developments in acoustics and the expanding capability of the application of acoustics to the 17 study of near-bed flow and sediment dynamics.

20 Key words

21 Waves; sediment transport; acoustics; boundary layer; bedload; ripples 
3 In dynamic marine environments, under the forcing of currents and waves, sediments are readily mobilised. Through the processes of accretion, erosion and transport, sediments define our coastal morphology, act as reservoirs for nutrients and contaminants, influence water quality and optical clarity, thereby impacting significantly on water chemistry and on primary production. Sediment dynamics in coastal waters therefore has relevance to a broad spectrum of marine science ranging from the physical and chemical, to the biological and ecological. In spite of the obvious importance of sediment transport, the predictive capability of models is still highly uncertain, particularly in the presence of bedforms and mixed sediments, therefore field measurements continue to be required for both site specific model tuning and validation (Davies et al, 2002; Davies and Thorne, 2008; Amoudry and Souza, 2011; Lu et al, 2015). It is also widely recognised that one of the components to improving large scale sediment transport modelling, requires accurate parameterisations of small scale sediment processes (Baumert et al, 2000; James, 2002; Ribberink et al, 2008; O’Hara Murray et al, 2012; Malarkey et al, 2015). At present however, quantification and understanding of detailed near-bed sediment transport processes is limited by the difficulty in obtaining co-located, non-intrusive, high spatialtemporal resolution measurements, of the dynamic feedback interactions between the bedforms, the hydrodynamics and the mobile sediments. The central problem is that sediment transport dynamics show complex variability over multiple temporal-spatial scales (Hay and Bowen, 1994; Villard and Osborne, 2002; Williams et al, 2003; Thorne et al, 2009; O'Hara Murray et al, 2011; Naqshband et al, 2014a, 2017; Moate et al, 2016), whilst most traditional measurement techniques provide only single point measurements.

To obtain measurements with high temporal-spatial resolution optical and acoustical instruments have been developed. Optical backscatter (Kineke and Sternberg, 1992; Bunt et al, 1999; Puleo et al, 2006; Rai and Kumar, 2015) and LISST, Laser In Situ Scattering Transmissometry, (Traykovski et al, 1999a; Agrawal et al, 2008; Graham et al, 2012; Agrawal and Hanes, 2015) are the more commonly used optical devices, however, they only provide information on suspended sediments at a single height above the bed. More recently Particle Image Velocimetry, PIV, has become a primary instrument for laboratory boundary layer sediment studies in the optical regime. This provides a Laser measuring techniques to obtain 
1 bedforms, suspended sediment concentration and velocity in two-dimensions, over a horizontal and vertical slice, 2DHV, with typical dimensions of respectively of $20 \mathrm{~cm} \times 10 \mathrm{~cm}$ 3 (Reidenbach et al, 2010; Liu and Lam, 2015; Malarkey et al, 2015; Yu and Xu, 2016). These instruments have proved very useful in laboratory studies, however, most PIV instruments have high energy consumption and are generally too cumbersome and intrusive for field deployment, although developmental autonomous instruments have been deployed (Wang et al, 2012; Wang and Liao, 2016). A further major drawback with PIV is that below or above an optimum number of particles in suspension the performance degrades due to insufficient scatterers in the former case and high optical attenuation in the latter case. Also imaging the bedforms during high suspension events becomes difficult. Therefore, under the more dynamically interesting conditions, when there are high concentrations of bedload and suspended load, the performance of PIV can become problematic (Lee et al, 2009).

A complementary approach to the optical systems are acoustic scattering based flow measuring techniques, which have received growing usage over the past three decades, due in part to a robust theoretical basis, their applicability in both laboratory and field conditions, the development of new technology, and the non-intrusive nature of the measurements (Thorne and Hanes, 2002). Acoustic instruments for measuring boundary layer sediment transport processes mainly fall into one of three categories: those which measure (i) bedforms (Hay, 2011; O’Hara Murray et al, 2012), (ii) profiles of suspended sediments (Thorne and Hurther, 2014) and (iii) profiles of the multi-component velocity field (Lhermitte and Lemmin, 1994; Stanton, 1996; Zedel et al, 1996; , Lemmin and Rolland, 1997; Hurther and Lemmin, 1998; Zedel and Hay, 1999; Hurther and Lemmin, 2000, 2001; Zedel and Hay, 2002; Hurther and Lemmin 2003; Hurther et al, 2007; Hurther and Lemmin, 2008; Hay et al, 2012). Here we look at developments and recent advances in these acoustic instruments in the context of a large flume facility deployment, which provided controlled laboratory conditions at natural flow scaling (Thorne and Hurther, 2017).

The acoustic measurements of small scale bed features have typically been obtained using sector scanning sonar technology, SSS, adapted for high resolution images of bedform morphology, or specifically developed acoustic ripple profilers, ARP, that provide a profile of the bed height along a transect. The high resolution SSS's normally have a frequency of around 1-2 $\mathrm{MHz}$, with beam widths of about $1^{\circ}$ in the azimuth and $30^{\circ}$ in elevation, rotate through $360^{\circ}$ in the horizontal and are mounted 1-2 $\mathrm{m}$ above the bed. As an acoustic pulse is 
1 backscattered from the bed, the envelope of the signal is measured and usually displayed as 2 image intensity. These bedform images are mainly used to categorise bed features, though quantitative estimates of bedform wavelength can obtained, and limited information on ripple height extracted (Hay and Wilson, 1994; Traykovski et al, 1999b; Williams et al, 2000; Hay, 2011). ARP's usually use a downward pointing narrow pencil beam pulse of sound around 1$2 \mathrm{MHz}$, mounted 1-2 $\mathrm{m}$ above the bed and radially rotates vertically through about $150^{\circ}$ to provide a digitised profile of the bed along a transect of around 3-5 $\mathrm{m}$. Both bedform height and wavelength can be obtained quantitatively (Bell and Thorne, 1997a, b; Bell et al, 1998; Thorne et al, 2003; William et al, 2005; O’Hara Murray et al, 2012; Larsen et al, 2015; Davies and Thorne, 2016). More recently combining the aerial coverage of the SSS with the quantitative measurements of the ARP the development of 3D-ARP's has taken place (Traykovski, 2007; Bell and Thorne, 2007; Thorne et al, 2013; Kramer and Winter, 2016; Moate et al, 2016). The 3D-ARP utilises ARP technology and collects transects as the pencil beam rotates horizontally through $180^{\circ}$ to provide high resolution quantitative three dimensional measurements of the bed micro-topography, which can be analysed for bedform dimensions and direction of mobility. It is the application of this latter, more recently developed instrument, which is the concern of the present study.

In the marine environment, most inorganic sand sized sediments have material densities and compressional sound velocities that are substantially greater than that of seawater, and therefore suspended sands are strong scatterers of underwater sound at $\mathrm{MHz}$ frequencies (Hay, 1991; Schaafsma and Hay, 1997; Thorne and Meral, 2008; Moate and Thorne, 2012). To exploit this principle, a number of monostatic multi-frequency Acoustic Backscatter Systems, ABS, have been developed to observe suspended sediments in the bottom 1-2 $\mathrm{m}$ above the seabed (Hess and Bedford, 1985; Crawford and Hay, 1993; Thorne and Hardcastle, 1997; Thorne et al, 2002; Dolphin and Vincent, 2009; Wilson and Hay, 2015a). Since their development, ABS have gained broad utility within sediment transport studies with a variety of observations, from coastal zone applications (Vincent et al, 1991; Hay and Bowden, 1994; Lee et al, 2004; Malarkey et al, 2015), and the dependence of sediment diffusivity on bedforms (Thorne et al, 2009), to sediment entrainment processes under irregular waves (O'Hara-Murray et al, 2011, 2012). Conventional ABS provide one dimensional in the vertical, 1DV, measurements of the bed location and suspended sediment profiles, however, it is difficult (Moate et al, 2011) using such ABS to simultaneously investigate the suspended sediment 
1 An array of closely spaced acoustic transducers would be able to provide observations of 2 bedforms and suspended sediments over a horizontal transect in the vertical providing two 3 dimensional horizontal and vertical, 2DHV, measurements of suspended sediment structures. 4 A multi-frequency array of 45 transducers, covering a $1.5 \mathrm{~m}$ transect has been developed 5 (Moate et al 2016) for collecting 2DHV data and has the acronym BASSI, the Bedform And 6 Suspended Sediment Imager. Here observations of near-bed suspended sediments collected using the BASSI are presented.

9 Advances in flow driven sediment transport physics and modelling still suffer from a lack of 10 high resolution flow measurement technologies providing process oriented data of turbulent 11 flow-particle interactions in the near-bed boundary layer. This is particularly the case in high 12 Reynolds number geophysical flows driven by energetic gravity currents in rivers or by surface gravity waves in the coastal marine environment. Referring to Heathershaw and Thorne (1985) over more than 30 years ago, Dyer and Soulsby (1988) recognized early the capabilities of acoustic instrumentation in process oriented studies of sediment transport because of their, at the time potential, ability to measure both flow velocity and sediment concentration at rates resolving the relevant turbulent flow scales. Since then, only few attempts were tested out in combining into a single instrument, the multi-frequency Acoustic Backscattering System, ABS, technology with the Acoustic Doppler Velocity Profiler, ADVP, technology. Shen and Lemmin (1999) designed a first version of an Acoustic Sediment Flux Profiler using simultaneously backscatter and forward scattering measurements for concentration measurements and co-located multi-bistatic pulse coherent velocity measurements. The system gave accurate results in terms of concentration, velocity and sediment flux profiling over a moveable sand layer of about $10 \mathrm{~cm}$ thick in highly turbulent open-channel flows (Cellino and Graf, 1999; Hurther and Lemmin, 2003). Nevertheless, the system required the vertical alignment of two emitting / receiving transducers mounted face-to-face. This induced local near-bed flow perturbation induced by the transducer placed into the sediment bed. Furthermore, the spatial and temporal resolutions were limited to $6 \mathrm{~mm}$ and $100 \mathrm{~ms}$, respectively, whereas for small scale turbulent flow, resolution closer to $1 \mathrm{~mm}$ and $10 \mathrm{~ms}$ are preferable. Zedel and Hay (1999) and Smyth et al (2002) also demonstrated the possibility of co-located velocity and concentration profiling with their acoustic Coherent Doppler Profiler. In this case, the system only allowed one component vertical velocity profiling which is of limited utility in the presence of two and three component wave and turbulent flow processes. 
1 Over recent years a novel acoustic measurement tool, the Acoustic Concentration and Velocity 2 Profiler, ACVP, (Hurther et al, 2011; Thorne et al, 2011; Thorne and Hurther, 2014; Wilson 3 and Hay, 2016) has been under development. Compared to previous sediment flux profilers, 4 the ACVP technology is not restricted to suspended particle flux profiling, but extends the 5 sediment flux profiling at turbulent flow scales into the dense bedload layer (Hurther and 6 Thorne, 2011; Naqshband et al, 2014b; Revil-Baudard et al, 2015, 2016; Fromant et al, 2017, 7 2018). In the present study, it is shown show how these nearbed ACVP measurement, are used 8 complementary with the BASSI and 3D-ARP data, to investigate wave-driven sand transport 9 processes over a rippled sand-bed.

11 The results in the present study formed part of the Joint Research Activities projects within the 12 European Hydralab IV programme, Water Interface Sediment Experiment, WISE, and ongoing 13 Hydralab+, http://hydralab.eu/, Cross disciplinary Observations of Morphodynamics and 14 Protective structures Linked to Ecology and eXtreme events, COMPLEX. 


\section{2 . Flume facility and instrumentation.}

2 The measurements were collected in the large-scale wave channel at UPC, Universitat 3 Politecnica de Catalunya, in Barcelona, Spain, http://ciemlab.upc.edu/en/facilities/ciem-1. The 4 dimensions of the flume are $100 \mathrm{~m}$ long, $3 \mathrm{~m}$ wide and $5 \mathrm{~m}$ deep and the wave paddle 5 characteristics at the deep water end of the channel were designed to establish nearly full-scale 6 gravity wave conditions. The measurement section was located between $55-58 \mathrm{~m}$ from the 7 wave paddle with a still water depth at $55 \mathrm{~m}$ equal to $\mathrm{h}=1.6 \mathrm{~m}$ above the sand-bed. The mean bed slope was initially levelled to a value of $1 / 15$, equivalent to $3.8^{\circ}$. The distance between the measurement section and the onshore located wave breaking region exceeded $10 \mathrm{~m}$ in order to limit the effect of breaking induced sediment transport in the measurement section. The bed consisted of well-sorted medium size quartz sand of median diameter, $\mathrm{d}_{50}=243 \mu \mathrm{m}$ and with a thickness of $0.9 \mathrm{~m}$ at the measurement location. Twelve acoustic and resistive wave gauges were distributed along the flume to track the time evolution of the surface elevation and to extract the water level statistics and the corresponding wave properties in the different nearshore regions. Runs of regular waves were generated over 20 minute long sequences containing 260 waves. The wave period, $\mathrm{T}$, heights, $\mathrm{H}$ and water depth, $\mathrm{h}_{\mathrm{o}}$, at the wave paddle were respectively set to $\mathrm{T}=4.5 \mathrm{~s}, \mathrm{H}=0.3-0.5 \mathrm{~m}$ and $\mathrm{h}_{0}=2.5 \mathrm{~m}$, respectively. The set of acoustic instrumentation consisted of a three dimensional Acoustic Ripple Profiler, 3D-ARP, a novel Bedform And Suspended Sediment Imager, BASSI, and three Acoustic Concentration and Velocity Profilers, ACVP. The 3D-ARP and BASSI were made to order following collaborative discussions on the design specifications by Marine Electronics, Guernsey, UK (http://www.marine-electronics.co.uk/) and the ACVP is a development instrument by LEGI, CNRS, France. The instruments were mounted on a metal pole frame which was constructed to be open in structure to minimise interference with the hydrodynamics and the bed, and yet sufficiently rigid to allow measurements to be made which were not impacted by induced vibrations due to the flow. Figure 1 provides a photograph of the arrangement and figures $2 \mathrm{a}$ and $2 b$ the configuration of the instruments relative to one another.

The 3D-ARP system is a self-contained battery powered autonomous dual axis mechanically scanning sonar underwater unit. The sonar has an oil-filled hemi-spherical plastic housing providing protection for its internally rotating transducer which operates at $1.1 \mathrm{MHz}$ with a narrow conical beam pattern. Typically, the sonar is mounted vertically, looking down at the 
1 bed, and captures a sequence of transects of the bed over a pre-programmed sector and range.

2 The sonar gathers a single swath of data in the horizontal plane and then rotates the transducer 3 through a pre-programmed angle around the vertical axis and repeats the process until a circular area underneath the sonar has been scanned in a sequence of radial transects. The stored raw binary data represents the amplitude of the envelope of the backscattered signal with logarithmic compression to achieve an overall dynamic range of greater than $90 \mathrm{~dB}$. The maximum resolution for the angular scanning and digitisation sampling of the backscattered envelope are respectively $0.9^{\circ}$ and $1.0 \mu \mathrm{s}$. The system is quite flexible and operates under software control which allows operating range, pulse length, sampling interval, swath arc and rotation angles to be operator selected. The 3D-ARP also has a collection of sensors to measure conductivity, temperature, depth, pitch and roll which are monitored for each dataset and stored in the data files. The profiles of the backscatter signal envelope are stored internally and post processed to extract the bed location to form transects along the bed which are combined to render a three dimension surface relief of the bed micro-topography.

The BASSI is a field deployable autonomous system consisting of three transducer line arrays, SN:001, SN:002 and SN:003, each of which was connected to a common electronic scheduling unit that controlled the sampling parameters. Each array contained 15 individual narrow beam disc transducers, consisting of five triple frequency groups $2.5,1.25$, and $0.75 \mathrm{MHz}$ evenly spaced along the array. The transducer arrays were designed to be compact, being $0.5 \mathrm{~m}$ long, $0.14 \mathrm{~m}$ wide and $0.07 \mathrm{~m}$ in depth, so as to minimise interference with the measured sediment processes. Each array was self-contained, housing the transmit/receive electronics and data storage capacity. In the present study the three transducer arrays were connected inline, and the complete system consisted of 45 transducers spaced regularly at $3.3 \mathrm{~cm}$ intervals over a $1.5 \mathrm{~m}$ range in the horizontal. To control the operation of the arrays the scheduling unit was employed to provided flexibility in the instrument setup. This allowed a selection of options for the sampling range, the sampling interval, the pulse length, the number of profiles averaged over and the pulse repetition frequency.

When deploying the BASSI, only one group of three frequency adjacent transducers were operational at once in a given line array, with sequential advancement along the array to the next group of three frequencies. Hence, five transmit/receive cycles were required to sample across the whole array. Following data capture, the data were converted to $16 \mathrm{bit}$, averaged over a selected number of successive transmissions, and written to internal USB flash drives. 
1 When multiple transducer arrays were connected to the scheduling unit, as was the case here, 2 the arrays electronically operated in parallel.

4 Three ACVP systems each composed of one emitter and two bistatic receivers were deployed in the measurement section. Shown in figure $1 \mathrm{~b}$ is the detailed configuration of the three

6 ACVP's. ACVP1 and ACVP2 were respectively located at distances of $22 \mathrm{~cm}$ and $28 \mathrm{~cm}$ above

7 the bed to perform velocity and sediment transport measurements above the water-bed 8 interface. However, due to their limitations of $3 \mathrm{~mm}$ vertical resolution with a temporal 9 resolution of $30 \mathrm{~ms}$, a third ACVP3 had a specific geometric configuration and electronic 10 hardware for profiling across the near-bed flow layers and into the bedload layer. ACVP3 was 11 located at $10 \mathrm{~cm}$ above the sand-bed and had a vertical and temporal resolution respectively of $121.5 \mathrm{~mm}$ and $30 \mathrm{~ms}$. In order to compare measurements obtained with the BASSI system, the 13 three ACVPs were positioned in the $1.5 \mathrm{~m}$ long measuring transect of the BASSI. Nevertheless, 14 the ACVPs were separated laterally from the BASSI by a distance of at least $20 \mathrm{~cm}$ to avoid acoustic interferences between the ACVP and the BASSI transmissions.

16 All three ACVP systems combined ADVP and ABS technologies as previously described in 17 Hurther et al (2011) and Thorne et al (2011). They provided one dimensional in the vertical 18 1DV, sediment concentration and two component, horizontal and vertical, velocity profiling at 19 a high spatial-temporal resolution allowing intra-wave and turbulence flow scales to be measured. In addition to these profiling abilities, the Acoustic Bed Interface Tracking, ABIT, method of Hurther and Thorne (2011) was used here to evaluate the vertical position of the undisturbed bed level. The lower suspension layer (equivalent to upper bedload interface) was defined as the height where the intra-wave concentration reached a value of $8 \%$ in volumetric sand concentration (Ribberink et al, 2008).

It was considered the combination of the 3D-ARP, BASSI and ACVP's would provide complementary and overlapping capabilities, thereby allowing some degree of intercomparison between instruments and also allowing a range of scales to be studied. 


\section{3 . Results}

2 In the present section the bedforms and the suspension dynamics are first examined on the basis

3 of the bed morphology measurements provided by the 3D-ARP and the time-resolved 2DHV suspension load obtained using the novel BASSI technology. Secondly the complementary time-resolved profiling of sand fluxes across the near-bed boundary layer are considered on the

6 basis of data collected by the ACVP.

\subsection{Bedform and suspension layer dynamics.}

\subsubsection{The three dimensional Acoustic Ripple Profiler, 3D-ARP, measurements.}

To examine the measurements from the acoustic instruments deployed in the large scale flume, the aim was to generate two dimensional steep ripples with heights of a few centimetres and wavelengths of a couple of decimetres. To obtain such ripples a number of predictors were used to calculate the wave conditions required to obtain the desired dimensions. Different predictors gave somewhat different dimensions, however, they were typically in the regime required, as illustrated by the predictor of Soulsby et al (2012) which for $\mathrm{H}=0.4 \mathrm{~m}, \mathrm{~T}=4.5 \mathrm{~s}$, $\mathrm{h}=1.6 \mathrm{~m}$ and $\mathrm{d}_{50}=243 \mu \mathrm{m}$ yields a ripple height, $\eta$, wavelength, $\lambda$, and slope respectively of $\eta=3.6 \mathrm{~cm}, \lambda=24 \mathrm{~cm}$ and $\eta / \lambda=0.15$.

The 3D-ARP was mounted $1.0 \mathrm{~m}$ above the bed and operated by collecting a series of transects of the bed as the instrument rotated horizontally through $180^{\circ}$ in $0.9^{\circ}$ step intervals to build up high spatial resolution three dimensional measurements of the bedform morphology. Digitisation of the backscattered signal envelope was $1 \mu$ s thereby providing a radial range resolution of $0.75 \mathrm{~mm}$. Previous studies with a precursor 2D-ARP (single transect) from the same manufacture (Bell and Thorne 1997a, b; Bell et al, 1998) compared direct transect measurements of a plaster caste replica of beach ripples with 2D-ARP measurements. The direct transects measurements yielded $\eta=1.7 \pm 0.2 \mathrm{~cm}$ and $\lambda=27 \pm 2.2 \mathrm{~cm}$ for the plaster caste surface, while the 2D-ARP measurements gave $\eta=1.5 \pm 0.2 \mathrm{~cm}$ and $\lambda=28 \pm 3.0 \mathrm{~cm}$. The ripple dimensions for the surface show very comparable results and indicate millimetric resolution and accuracy for the 2D and 3D ARP's. To illustrate the operation of the 3D-ARP, single transects from the instrument are shown in figure 3. In figure 3a a transect is shown when waves had been propagating over the bed for 20 minutes and the wave generator switched off, 
1 the 3D-ARP was therefore operating in effectively clear water. It can be seen in the figure that

2 the interface between the bed and the water has been clearly identified as indicated by the white

3 line and a representative transect of the bed obtained. The underlying slope was due to the 1/15 gradient of the bed and not due to the instrument. Two hundred such transects were obtained for each measurement. Using data from another experiment, the more difficult, though obviously necessary task, of identifying the interface under conditions when waves were propagating and instantaneous concentration reached values of the order of $10 \mathrm{kgm}^{-3}$, is presented in figure $3 \mathrm{~b}$. As shown in the image, although significant suspension was present, identification of the sediment-water interface was still successfully obtained. To assess the measurement for the case shown in figure $3 \mathrm{~b}$ all 200 transects were analysed yielding $\eta=3.5 \pm 0.3 \mathrm{~cm}, \lambda=49.8 \pm 11 \mathrm{~cm}$. This compares well with the mean values from all other $\mathrm{H}=0.5$ $m$ clear water cases which gave averaged values of $\bar{\eta}=3.9 \pm 0.5 \mathrm{~cm}, \bar{\lambda}=46 \pm 4 \mathrm{~cm}$. Therefore there was no significant difference between the case with suspended sediments present and the clear water cases. This supports the veracity of the 3D-ARP to obtain accurate bedform measurements under dynamic conditions of high suspended load.

As mentioned above the expectation had been that two dimension steep ripples orthogonal to the direction of wave propagation, would be generated in the large scale flume. In figure 4a the bedform morphology constructed from the 200 profile transects is shown for the case of $\mathrm{H}=0.5$ $\mathrm{m}$. The vertical structure to the right hand side of the figure is a reflection from the side wall of the flume, due to the mounting of the instrumentation not being centred in the flume. This image is typical of all the bedform measurements collected with the 3D-ARP and clearly shows that the bedforms were not as anticipate, two dimension ripples, but much more irregular and three dimensional in form. Figure $4 \mathrm{~b}$ shows an analysis of the bedform dimensions for the individual transects over the $180^{\circ}$ rotation for $\mathrm{H}=0.4 \mathrm{~m}$. For the analysis the ripple height was calculated using $\eta=2 \sqrt{2} \zeta_{\text {rms }}$ where $\zeta_{\text {rms }}$ was the root-mean-square height of the transect profile and the ripple wavelength was obtained using $\lambda=2 \bar{\xi}$ where $\bar{\xi}$ was the mean zero crossing distance over the profile. The mean bed slope was removed prior to analysis. The analysis shows comparable ripple dimensions throughout the $180^{\circ}$ rotation, thereby identifying the three dimensionality of the bed morphology. Such images as those shown in figure $4 \mathrm{a}$ and the analysis in figure $4 \mathrm{~b}$ illustrate the advantage of the 3D-ARP over a 2D-ARP, where it would not have been as straightforward to assess that the cross-flume variation in morphology was closer to three dimensional in structure, rather than two dimensional. As an independent check 
1 on the acoustic measurements, plan and side view photographs of the bed were collected and are shown in figure 4c. These observations are consistent with the scale and morphology of the bedforms measured acoustically.

To assess the consistency of the measured ripple dimensions with the 3D-ARP a number of repeat experiments were carried out at different wave heights. The results are shown in figure 5 for $\mathrm{H}=0.3 \mathrm{~m}, 0.4 \mathrm{~m}$ and $0.5 \mathrm{~m}$. The results are seen to be consistent, with the average ripple dimensions for $\mathrm{H}=0.3 \mathrm{~m}$ having values of $\eta=1.8 \pm 0.1 \mathrm{~cm}, \lambda=22 \pm 1.8 \mathrm{~cm}, \eta / \lambda=0.079 \pm 0.008$, for $\mathrm{H}=0.4 \mathrm{~m}$ of $\eta=2.4 \pm 0.2 \mathrm{~cm}, \lambda=29 \pm 1.8 \mathrm{~cm}, \eta / \lambda=0.08 \pm 0.008$ and for $\mathrm{H}=0.5 \mathrm{~m}$ of $\eta=3.9 \pm 0.5 \mathrm{~cm}$, $\lambda=46 \pm 3.7 \mathrm{~cm}, \eta / \lambda=0.084 \pm 0.012$. The ratio of the standard deviation to the mean for the whole data set for $\sigma(\eta) / \eta, \sigma(\lambda) / \lambda$, and $\sigma(\eta / \lambda) /(\eta / \lambda)$ respectively had values of $0.09 \pm 0.04,0.08 \pm 0.01$ and $0.11 \pm 0.03$. Given that there will be some reconfiguration of the bed during the repeat experiments these result provide evidence for the repeatability of the 3D-ARP to accurately measure ripple dimensions. Another approach to looking at consistency was to compare adjacent transects $0.9^{\circ}$ apart. Given the decimetric horizontal scale of the bedforms the expectation was that adjacent profiles would be very comparable. To carry out the analyse the parameter $\delta / \eta$ was calculated, where $\delta=\overline{\left|t_{h_{j}}-t_{h_{1}}\right|}$, $t_{h}$ was the profile heights along a transect, subscripts $\mathrm{i}$, $\mathrm{j}$ were adjacent transects and the over bar represents an average over all the transects for an experimental run. The results are shown in $5 \mathrm{~d}$. The mean values for $\delta / \eta$ over all experimental runs for $\mathrm{H}=0.3 \mathrm{~m}, 0.4 \mathrm{~m}$ and $0.5 \mathrm{~m}$ was respectively $0.13,0.07$ and 0.07 , which using the mean ripple heights for the three wave conditions yields a value for $\delta \approx 2 \mathrm{~mm}$. Given that adjacent transect profiles are not identical this estimate is considered a reasonable estimate of repeatability and accuracy which is consistent with previous estimates (Bell and Thorne, 1997a, b; Bell et al, 1998) noted earlier in this section.

Although it was not the purpose of the present study to assess the accuracy of ripple predictors under waves, the aim was to look at the performance of the 3D-ARP, it was still considered of interest to see how the observations compared with the predictions used to design the experimental study. To this end Soulsby et al (2012) and Goldstein et al (2013), both of which are based on large complied data sets, have been used as representative predictors. The Soulsby et al (2012) formulation is given by

$$
\frac{\lambda}{A}=\left[1+0.00187 \frac{A}{d_{50}}\left(1-e^{-\left(0.0002 \frac{A}{d_{50}}\right)^{1.5}}\right)\right]^{-1}
$$




$$
\frac{\eta}{\lambda}=0.15\left[1-\mathrm{e}^{-\left(5000 \frac{\mathrm{A}}{\mathrm{d}_{50}}\right)^{3.5}}\right]
$$

$$
\frac{\eta}{\mathrm{A}}=\frac{\lambda}{\mathrm{A}} \frac{\eta}{\lambda}
$$

6 Goldstein et al 2013 can be expressed as,

$$
\frac{\lambda}{A}=\frac{2}{\left(1.12+2180 d_{50}\right)}
$$

$$
\frac{\eta}{A}=\frac{626 d_{50}}{1.12+2180 d_{50}}
$$

$$
\frac{\eta}{\lambda}=\frac{3.42}{22+\left(\frac{2 \mathrm{~A} /\left(1.12+2180 \mathrm{~d}_{50}\right)}{1000 \mathrm{~d}_{50}}\right)^{2}}
$$

$12 \mathrm{~A}$ is the orbital wave amplitude at the bed and $\mathrm{d}_{50}$ is the median diameter of the bed sediments.

13 A comparison of the predictors with the 3D-ARP measurements are presented in figure 6.

14 Soulsby et al 2012 tends to overestimate ripple height and underestimate ripple wavelength,

15 while Goldstein et al 2013 is in somewhat closer agreement with the observations, with an

16 invariant value for both ripple dimensions as $\mathrm{A}$ is varied for fixed $\mathrm{d}_{50}$. Both predictors estimate

17 a ripple slope of about 0.15 , while the observed slope was closer to 0.1 , the lower observed

18 slope may be associated with the three dimensional nature of the bedforms.

Although no detailed analysis of the flume was carried out to try and ascertain why three dimensional bedforms were formed, the bed had a gradient of $1 / 15$ and there were wave reflection from the beach which added to the complexity of the surface waveforms in the measurement area. Further the medium sized sand may have become mobile by small, low energy, three-dimensional turbulent flow eddies as observed by O'Donoghue et al (2006). All 
1 of which coupled with the influence of the side walls of the flume, may have led to the three 2 dimensionality of the bedforms.

3

\subsubsection{The Bedform And Suspended Sediment Imager, BASSI, measurements.}

5

6 The BASSI consisted of three line arrays, SN:001, SN:002 and SN:003, with each array 7 consisting of five sets of three frequency transducers, $0.75,1.25$ and $2.5 \mathrm{MHz}$. The instrument was mounted at a nominal height of $0.75 \mathrm{~m}$ above the bed, with a vertical resolution of $0.5 \mathrm{~cm}$ and covering a transect of $1.5 \mathrm{~m}$ and provided profiles averaged over 8 transmissions and resulted in $6 \mathrm{~Hz}$ measurements of the suspended sediments and bedforms over a transect. To obtained the suspended sediment concentration, $\mathrm{C}$, the following expression was used (Thorne and Hurther, 2014)

$$
\mathrm{C}=\left(\frac{\mathrm{r} \psi}{\mathrm{K} \Re}\right)^{2} \mathrm{~V}_{\mathrm{m}}^{2} \mathrm{e}^{4\left(\mathrm{r} \alpha_{\mathrm{w}}+\alpha_{\mathrm{s}}\right)}
$$

14 where

$$
K=\frac{f\left(x_{0}\right)}{\sqrt{a_{0} \rho}}, \quad \alpha_{s}=\int_{0}^{r} \xi C d r, \quad \xi=\frac{3 \chi\left(x_{o}\right)}{4 a_{o} \rho}
$$

with

$$
f\left(x_{0}\right)=\left[\frac{\int_{0}^{\infty} a n(a) d a \int_{0}^{\infty} a^{2} f_{i}(x)^{2} n(a) d a}{\int_{0}^{\infty} a^{3} n(a) d a}\right]^{1 / 2}
$$

$$
\chi\left(x_{0}\right)=\frac{\int_{0}^{\infty} a n(a) d a \int_{0}^{\infty} a^{2} \chi_{i}(x) n(a) d a}{\int_{0}^{\infty} a^{3} n(a) d a}
$$

$$
a_{o}=\int_{0}^{\infty} a n(a) d a
$$

and 


$$
f_{i}(x)=\frac{\left(1-0.35 \mathrm{e}^{-((x-1.5) / 0.35)^{2}}\right)\left(1+0.5 \mathrm{e}^{-((\mathrm{x}-1.8) / 2.2)^{2}}\right) \mathrm{x}^{2}}{1+0.9 \mathrm{x}^{2}}
$$

$$
\chi_{\mathrm{i}}(\mathrm{x})=\frac{0.29 \mathrm{x}^{4}}{0.95+1.28 \mathrm{x}^{2}+0.25 \mathrm{x}^{4}}
$$

$4 \quad V_{\mathrm{m}}^{2}$ is the mean square recorded backscatter signal, $\mathrm{r}$ is the range from the transceiver, $\psi$ accounts for the departure from spherical spreading within the transducer nearfield (Downing et al, 1995) and $\Re$ is the system constant (Betteridge et al, 2008). The value for $\Re$ was calculated for each transducer by comparing the output from the BASSI with a calibrated ABS in a combined field deployment. Details of this calibration and its outcome are presented in Moate et al (2016). K represents the sediment backscattering properties, $\rho$ is the sediment grain density and $a_{0}$ is the suspension mean particle radius. The term $\alpha_{\omega}$ is the sound attenuation due to water absorption and $\alpha_{\mathrm{s}}$ is the attenuation due to suspended sediment scattering. $\mathrm{f}_{\mathrm{i}}(\mathrm{x})$ and $\chi_{i}(x)$ are respectively the intrinsic form function and intrinsic normalised total scattering crosssection for the particles in suspension (Thorne and Meral, 2008) and $\mathrm{x}=\mathrm{ka}$, where $\mathrm{k}$ is the wavenumber of the sound and $\mathrm{a}$ is the radii of the particles in suspension. $\mathrm{f}\left(\mathrm{x}_{\mathrm{o}}\right)$ and $\chi\left(\mathrm{x}_{0}\right)$ represent the ensemble mean scattering values obtained by integrating the intrinsic scattering characteristics over the particle size probability density function, $n(a)$, of the particles in suspension and $\mathrm{x}_{0}=\mathrm{ka}_{0}$. To obtain values for $\mathrm{a}_{0}, \mathrm{f}\left(\mathrm{x}_{0}\right)$ and $\chi\left(\mathrm{x}_{0}\right)$ the size probability density function for the bed sediments, $\mathrm{n}_{\mathrm{b}}(\mathrm{a})$, was measured and this was translated to $\mathrm{n}(\mathrm{a})$ following Davies and Thorne (2016). $\mathrm{f}\left(\mathrm{x}_{\mathrm{o}}\right)$ and $\chi\left(\mathrm{x}_{0}\right)$ were evaluated at the three BASSI frequencies and used in an inversion (Thorne and Hurther, 2014) to calculate the suspended sediment concentration.

The main objective for developing the BASSI was to be able to image suspended sediment structures over bedforms and thereby contribute to a better understanding of boundary layer sediment dynamics. In the first instance an internal check was carried out to ascertain if the different frequencies yielded comparable concentrations. Figure 7 shows the near-bed time varying suspended concentration field measured at the three different frequency under regular waves of amplitude $\mathrm{H}=0.5 \mathrm{~m}$ and period $\mathrm{T}=4.5 \mathrm{~s}$ for array $\mathrm{SN}$ :001. As can be seen the temporal variations of the concentration are comparable both in magnitude and structure and the signature of a repeatable pattern due to the presence of regular waves as the main driving force 
1 is observed. Following on from the comparison between individual transducers, the temporal

2 variation in concentration was obtained by averaging over the 15 transducers in each array. The

3 results for the three arrays are shown in figure 8 where again it can be seen that the magnitude, structures and repeatable pattern is observed. There are detailed differences in the suspended sediments between the arrays and these are likely associated with the three dimensionality of

6 the bedforms and the location of the arrays relative to the bedforms. To provide direct quantitative comparison of the measured suspended sediments, concentrations profiles averaged over an experiment, which typically had a duration of 20 minutes, was calculated for each frequency for each array. The results are shown in figure 9 and it can be clearly seen that the near-bed concentration profiles are comparable for the three frequencies in each array and the magnitude and form of the suspended sediment profiles are consistent across the three arrays. The results presented in figures 7-9 are indicative of the concentrations measured by the BASSI and support the capability of the instrument to obtain consistent concentrations over the three frequencies and across the 45 transducers that compose the BASSI.

Given the internal consistency of the BASSI analysis above, an example is presented in figure 10 of a two dimensional vertical and horizontal, $2 \mathrm{DHV}$, suspended sediment image generated by the BASSI. This shows a quasi-instantaneous (each recorded frame is a hardware average of 8 frames to reduce backscatter statistical configuration noise, Thorne and Hurther, 2014) image of the bed and suspended sediments. Collection of an image over the length of the array required $80 \mathrm{~ms}$. The structure of the suspension is relatively complex due to the interactions of the wave hydrodynamics with the three dimensional bedforms. The image is also somewhat noisy due to backscatter statistical noise. To obtain some context for the image presented in figure 10 a series of images $0.5 \mathrm{~s}$ apart are shown in figure 11. It can be clearly seen in images $11 \mathrm{a}-11 \mathrm{~d}$ that the main structures are moving from right to left in the figure, while in $11 \mathrm{e}-11 \mathrm{~h}$ following flow reversal the structures in the image move from left to right. The images in figure 11 show the capability of the BASSI to measure the time varying complex suspension field over a transect and therefore provides new opportunities for studying boundary layer sediment dynamics.

Using the BASSI as a more conventional ABS system, the concentration profiles from the 45 transducers were averaged to provide ensemble wave and ripple averaged profiles over a period of 20 minutes for three wave conditions, $\mathrm{H}=0.3 \mathrm{~m}, 0.4 \mathrm{~m}$ and $0.5 \mathrm{~m}$. The results with standard 
1 error bars are shown in figure 12. In the figures it can be seen that in the near-bed regime, 2 within approximately $0.1 \mathrm{~m}$ of the bed, the logarithmic concentration above the bed is linear 3 with height, while above $0.1 \mathrm{~m}$ the logarithmic concentration reduces at a slower rate than 4 linear. This observation is consistent for the three wave heights. The deviation in the general 5 trend in the profiles just below $\mathrm{z}=0.4 \mathrm{~m}$, which is most prominent in figure $12 \mathrm{a}$, was considered 6 to be due to an echo from the mounting arrangement for the instruments As with the bedforms, 7 it was considered of use to compare the present data with commonly used expressions for 8 predicting suspended concentration profile above bedforms. Two predictors were used those 9 of Nielsen (1992) and a power law based on the Rouse parameter (Rouse, 1937). The Nielsen 10 expression is

$$
\frac{\mathrm{C}(\mathrm{z})}{\mathrm{C}_{\mathrm{o}}}=\mathrm{e}^{-\mathrm{z} / \mathrm{L}_{\mathrm{s}}}
$$

where

$$
\begin{array}{ll}
L_{s}=0.075 \frac{A \omega}{w_{s}} \eta & \text { for } \frac{A \omega}{w_{s}}<18 \\
L_{s}=1.4 \eta & \text { for } \frac{A \omega}{w_{s}}<18
\end{array}
$$

$16 \mathrm{C}_{\mathrm{o}}$ is the reference concentration at $\mathrm{z}=0$ which is at the crest of the bedforms, $\mathrm{L}_{\mathrm{s}}$ is the vertical 17 mixing length, $\omega$ is the surface wave angular frequency, $\mathrm{w}_{\mathrm{s}}$ is the settling velocity based as 18 normally on $\mathrm{d}_{50}$ of the bed and $\eta$ is the averaged ripple height measured using the 3D-ARP and 19 shown in figure 5. The power law is given as (Hardisty, 1990; Soulsby, 1997)

$$
\frac{\mathrm{C}(\mathrm{z})}{\mathrm{C}_{\mathrm{a}}}=\left(\frac{\mathrm{z}}{\mathrm{z}_{\mathrm{a}}}\right)^{\frac{-\mathrm{w}_{\mathrm{s}}}{\beta \kappa \mathrm{u}_{*}}}
$$

where

$$
u_{*}=\sqrt{\frac{f_{w}}{2}} A \omega \quad, f_{w}=1.39\left(\frac{A}{\eta^{2} / \lambda}\right)^{-0.52}
$$


$1 \quad \mathrm{C}_{\mathrm{a}}$ is the reference concentration at reference height $\mathrm{z}_{\mathrm{a}}, \mathrm{z}_{\mathrm{a}}=0.5 \mathrm{~cm}, \beta$ was set to $0.8, \mathrm{u} *$ is the 2 form drag friction velocity, $f_{w}$ the wave friction factor and $\eta$ and $\lambda$ the averaged ripple height and wavelength respectively obtained from the 3D-ARP data shown in figure 5.

It can be seen that in the near-bed region, below $\mathrm{z} \approx 0.1 \mathrm{~m}$, Nielsen's exponential formulation and the exponential fitted line are consistent with the form of the observations, with somewhat improving correspondence between Nielsen's formulation and the data as the wave height increased. Alternatively above $\mathrm{z} \approx 0.1 \mathrm{~m}$ the Nielsen's expression departs significantly from the data and the Rouse power law provides a form for the concentration profile which is more consistent with the observations. The exponential and power law profiles are associated respectively with constant and linearly increasing sediment diffusivity profiles with height above the bed. These diffusivity profiles have been interpreted by Thorne et al (2009) as respectively due to convective near-bed entrainment, linked to intermittent formation of sediment laden vortices, which advect away from the bed, break up, leading to turbulent diffusion processes becoming more dominant further away from the bed. The suspended sediments profiles above the complex three dimensional ripples in the large scale flume, are therefore considered to arise from convective and diffusive process, with the former prevailing below $\mathrm{z} \approx 0.1 \mathrm{~m}$ and with diffusion becoming increasingly dominant above this height.

The BASSI was designed to obtain suspended sediments and bedforms over a transect. Therefore, over the $1.5 \mathrm{~m}$ cross-shore length of the BASSI the bed location was identified for each of the 45 transducers with a resolution of $0.005 \mathrm{~m}$ in the vertical and $0.033 \mathrm{~m}$ in the horizontal. As noted above, the BASSI provided averaged profiles at $6 \mathrm{~Hz}$ and 40 of these 6 $\mathrm{Hz}$ profiles were averaged over to yield $0.15 \mathrm{~Hz}$ bedform transect measurements. Averaging over the 40 profiles improved the robustness of the bed detection algorithm. A typical result is presented in figure 13a, which shows an intensity plot of the backscattered signal from the 45 transducers with a white line identifying the location of the bed. For this particular case $\mathrm{H}=0.4$ $\mathrm{m}$. In figure $13 \mathrm{~b}$ the evolution of the bedform transects over $1000 \mathrm{~s}$ is shown and the transects are seen to be coherent over time. In figure 13c the ripple dimensions over the $1000 \mathrm{~s}$ are presented and these are observed to be relatively uniform over the period.

In figure 14 BASSI ripple dimensions from experiments repeated with the same wave height and period are presented, with the results being analogous to those in figure 5 from the 3D- 
1 instruments. The average ripple dimensions from the BASSI for $\mathrm{H}=0.3 \mathrm{~m}$ had values of $\eta=2.4 \pm 0.1 \mathrm{~cm}, \lambda=24 \pm 2 \mathrm{~cm}, \eta / \lambda=0.1 \pm 0.009$, for $H=0.4 \mathrm{~m}$ of $\eta=2.9 \pm 0.14 \mathrm{~cm}, \lambda=27 \pm 3 \mathrm{~cm}$, $\eta / \lambda=0.11 \pm 0.006$ and for $H=0.5 \mathrm{~m}$ of $\eta=2.8 \pm 0.2 \mathrm{~cm}, \lambda=40 \pm 6 \mathrm{~cm}, \eta / \lambda=0.07 \pm 0.01$. These results are very comparable with those from the $3 \mathrm{D}$-ARP, though with the ripple heights showing some diverge. Given that the 3D-ARP measurements were of a $3 \mathrm{~m}$ diameter area of the bed normally collected at the end of an experiment with the waves switched off, suspended sediment levels very low and the bed immobile, while the BASSI measurements were of a single cross-shore transect collected throughout an experiment with high levels of suspended sediment, it is not unexpected that the ripple dimensions from both instruments would not be identical, particularly with regard to the sensitive measurement of ripple height. It is therefore considered that the results from both instruments are consistent within their different methodologies of data collection.

3.2 Near-bed boundary layer measurements using the Acoustic Concentration and Velocity Profiler, ACVP.

As shown in figure 12 the BASSI can provide profiles of the suspended sediment concentration with a vertical resolution of $0.5 \mathrm{~cm}$. However, to study the near-bed interface flow and sediment transport processes, higher spatial and temporal resolutions are required. To illustrate the advantages of bring complementary instrumentation together, in figure 15 comparative profiles of the time-averaged sand concentration from the BASSI and the ACVP are presented. This figure and all the following figures were collected for $\mathrm{H}=0.5 \mathrm{~m}$. As can be seen in figure 15 the BASSI provides the broad structure of the suspension profile up to $0.5 \mathrm{~m}$ above the rippled bed, while the ACVP provides much greater detail over the first $5 \mathrm{~cm}$ above the bed with a vertical resolution of $1.5 \mathrm{~mm}$. Combining the observations provides the full $2 \mathrm{DHV}$ outer suspension and the near-bed 1DV flow and sediment details for studying the dynamics of nearbed sediment processes.

The time-resolved 1DV profiling ability of the ACVP technology is applied to investigate the near-bed interface flow and sand transport processes in the present wave-driven ripple regime. In the following, the flow and sand transport are first examined in terms of mean wave and current velocity, sand concentration and sand flux measurements. In the second subsection, the intra-wave profile dynamics spatially averaged over one entire ripple profile (in the cross-shore direction), is considered for both suspension and bedload layers. In the third subsection, the 
1 spatial flow dynamics along a ripple profile is used to identify the presence of ripple vortices on either sides of the ripple crest defined as the offshore stoss and onshore lee sides. How the velocity, the concentration and the sand transport rate are spatially affected by the presence of ripple vortices is examined. Making full use of the ACVP time-resolved profiling ability at turbulent flow scales, the mean and intra-wave dynamics of sand transport rate are decomposed into their wave, current and turbulence driven components. These decomposed measurements permit the direct estimation of the suspended sand erosion and sand settling processes. Finally, a direct estimation of convective and turbulent sediment diffusivity profiles are derived, which are compared with the total sand diffusivity obtained from the mean sand settling flux and mean suspended concentration profiles.

Figure 16 shows the ACVP2 measured wave velocity, $\mathrm{u}_{\mathrm{w}}$ at the free-stream height defined at $10 \mathrm{~cm}$ above the ripple crest. $\mathrm{u}_{\mathrm{w}}=\langle\mathrm{u}(\mathrm{t} / \mathrm{T})>-\overline{\mathrm{u}(\mathrm{t})}$, where $\mathrm{u}(\mathrm{t})$ is the cross-shore velocity, $\mathrm{T}$ is the wave period and $\langle>$ and the overbar respectively represents ensemble wave phase averaged cross-shore velocity and the temporal mean velocity over 144 consecutive wave cycles. The free-stream height is located well above the top of the near-bed boundary layer which is taken as the elevation of the maximum overshoot velocity in the profile, presented in figure $17 \mathrm{a}$, of the root-mean-square wave velocity, $u_{\mathrm{rms}}$. As can be seen in figure 16 the free-stream wave shape reveals a strong positive velocity skewness with a value $S k=<u_{w}^{3}>/ u_{r m s}^{3}=0.6$, where $u_{\mathrm{rms}}=\sqrt{\left\langle\mathrm{u}_{\mathrm{w}}^{2}\right\rangle}$, due to the higher velocity amplitude during the wave crest, $0.64 \mathrm{~ms}^{-}$ ${ }^{1}$, compared to the lower value during the wave trough, $0.4 \mathrm{~ms}^{-1}$. Furthermore, a weak positive acceleration skewness Asy $=-\mathcal{H}<\mathrm{u}_{\mathrm{w}}{ }^{3}>/ \mathrm{u}_{\mathrm{rms}}^{3}=0.25$ is observed due to the stronger wave acceleration phase at $0.82<T<0.17$ than the deceleration phase for $0.17<t / T<0.82$. Here $\mathcal{H}$ refers to the Hilbert transform of the wave velocity component. These values are representative of surface-gravity waves propagating in the shallow coastal shoaling zone before the wave breaking zone.

\subsubsection{Mean flow and sand transport characteristics}

Also shown in Figure $17 \mathrm{~b}, 17 \mathrm{c}$ and $17 \mathrm{~d}$ are the profiles of mean current, $\overline{\mathrm{u}(\mathrm{t})}$, mean normalised sand concentration, $\overline{\mathrm{C}(\mathrm{t})} / \overline{\mathrm{C}(\mathrm{t})_{\mathrm{o}}}$, where $\mathrm{C}(\mathrm{t})_{\mathrm{o}}$ is the concentration at the ripple crest, and mean sand transport rate in the cross-shore direction calculated as the time-averaged product of the time-resolved velocity, $\mathrm{u}(\mathrm{t})$ and concentration, $\mathrm{C}(\mathrm{t})$, that is $\overline{\mathrm{u}(\mathrm{t}) \mathrm{C}(\mathrm{t})}$. Because both quantities 
1 of transport were measured at rates resolving the small turbulent flow scales, simultaneously

2 and in the same measurement volume, this time-averaged quantity represents the total sand transport rate (i.e. the sum of all contributing terms which are analysed later in the text).

The vertical axis in the profiles of all the following figures presented are represented as a function of the distance from the ripple crest taken as the origin of the vertical axis and generally normalized by the bed roughness height $\mathrm{k}_{s}$. The latter is approximated using the formulation of Nielsen (1992) modified by Thorne et al (2002) as $\mathrm{k}_{\mathrm{s}}=25 \eta^{2} / \lambda$ in order to take into account convective sand diffusion processes in the ripple regime. The values of ripple height $\eta$ and length $\lambda$ were obtained from the 3D-ARP measurements in figure 5 for the case of a wave height $\mathrm{H}=0.5 \mathrm{~m}$. Figure $17 \mathrm{~b}$ shows a weak offshore oriented current of about $-7 \mathrm{cms}^{-}$ 1 , this compares with the maximum undertow current of $-25 \mathrm{cms}^{-1}$ reached in the onshore located outer surfzone (not shown here), thereby indicating that the present measurements are taken offshore of the wave breaking zone. The mean sand concentration profile in Figure 17c is normalized by the $\mathrm{C}_{0}$ value measured at the ripple crest. The profile shows a strong increase in mean concentration for $\mathrm{z} / \mathrm{k}_{\mathrm{s}}<0.1(\mathrm{z}<\sim 8 \mathrm{~mm})$ above the ripple crest associated with the bedload layer. Above this layer the profile follows an exponential decay in the suspension layer. The total cross-shore sand flux profile shows a net onshore directed bed-load transport followed by a fully offshore directed flux in the suspension layer.

\subsubsection{Intra-wave dynamics}

Unlike the time-averaged measurements shown in figure 17, the intra-wave dynamics in figure 18 are analysed on the basis of an ensemble phase average over 144 consecutive waves versus $\mathrm{t} / \mathrm{T}$. This represents a total duration of more than ten minutes which was needed for an entire ripple to migrate beneath the ACVP sample volume in the onshore direction. The ripple migration direction was determined from the BASSI bed shape evolution measurements. As a consequence, both the time-averaged and intra-wave measurements represented in figures 17 and 18 , respectively, are spatially averaged over an entire ripple length.

Figure 18 presents the intra-wave dynamics of a) the cross-shore free-stream wave velocity, b) the cross-shore wave velocity profiles, c) the relative sand concentration profiles and d) the cross-shore sand flux profiles. The thick solid black line in figures $18 \mathrm{~b}$ and $18 \mathrm{~d}$ shows the 
1 interface separating the lower bedload region from the upper suspension layer (taken as the 2 height of volumetric sand concentration equal to $8 \%$ as commonly proposed in Ribberink et al. 3 2008). The dashed white lines in figure $18 \mathrm{c}$ refer to the fixed heights of the intra-wave concentrations represented in figures $18 \mathrm{e}$ and $18 \mathrm{f}$ in the bedload and suspension layers, respectively. Inside the bedload layer figure 18e shows that under the wave crest as the undisturbed bed is approached, that is from the lowest to highest relative concentration, there is a progressive increase in the phase lead. As seen in figure 17a, this region is associated with a strong vertical gradient in wave velocity magnitude induced by the local bed friction inside the bedload layer.

In figure $18 \mathrm{f}$ the relative suspended concentration with height above the bedload layer is shown, with the highest concentration being closest to the bedload layer. In contrast to the intra-wave dynamics inside the bedload layer, the concentration time-series present a much stronger intrawave variability. In particular during the wave trough, two local maxima are detected over an extended vertical region up to nominally $\mathrm{z} / \mathrm{k}_{\mathrm{s}}=1(\mathrm{z} \sim 8 \mathrm{~cm})$, above the ripple crest. This might be the signature of a ripple vortex in the suspension layer involving a phase coherent intrawave velocity variation at a temporal scale smaller than the wave period. Figure $18 \mathrm{~d}$ reveals that this signature can also be identified in the suspended sand flux field. In contrast to the bedload layer, there is negligible phase shift between the relative concentration time series in the suspension layer which suggests a depth homogeneous flow structures present during the wave trough half-cycle.

\subsubsection{Spatial dynamics along a ripple profile}

Figures 19a-19e respectively presents the time-averaged cross-shore velocity, $\mathrm{u}$, the magnitude of the cross-shore velocity, $|u|$, the magnitude of the vertical wave velocity, $|w|$, the logarithmic concentration and the cross-shore sand flux. Each measurement was averaged over four consecutive wave periods for each location above the bed along a ripple profile. This was measured with the ACVP2 over a vertical profiling range of $-1<\mathrm{z} / \eta<3$ (or $-0.5<\mathrm{z} / \mathrm{k}_{\mathrm{s}}<1.5$ ) and a total duration of 144 wave periods. The $\mathrm{x}$-axis in figures 19 was obtained by assuming a constant ripple migration speed during the onshore migration of an entire ripple profile beneath the vertical ultrasound beam of ACVP2. Also represented in all figures 19, are line profiles of the corresponding quantity at equally spaced locations along the ripple profile. The undisturbed 
1 bed level represented by the thick solid black line in all the panels in figure 19 was obtained 2 with the ABIT method proposed by Hurther and Thorne (2011). This reveals the presence of an asymmetrical ripple shape typical of sand ripples under velocity skewed oscillatory flows (van der Werf et al, 2007) or waves (Hurther and Thorne, 2011), composed of a milder bed slope along the large ripple stoss face relative to the steeper and shorter lee face of the ripple.

Figure 19a corresponds to a colour plot of the time-averaged velocity as the streaming velocity component above the onshore migrating sand ripple. Inside the near-bed boundary layer, for $\mathrm{z} / \eta_{\mathrm{s}}<0.3$ (or $\mathrm{z} / \mathrm{k}_{\mathrm{s}}<0.15$ ), the red zone along the ripple profile shows an onshore velocity streaming spatially homogeneous along the stoss face of the ripple and with a local maximum on the lee face at $x / \lambda \cong 0.75$. Above the near-bed boundary layer the mean velocity $u$ is offshore oriented all along the ripple profile and with a maximum value of about $-0.1 \mathrm{~ms}^{-1}$ above the stoss face of the ripple at $\mathrm{x} / \lambda \cong 0.4$. The most noticeable evidence of a ripple vortex is seen in the strong spatial inhomogeneity of all velocity data (figures 19a, 19b,19c) on the lee face of the ripple above the near-bed boundary layer, for $-0.5<\mathrm{z} / \eta<2$. In particular the mean uvelocity, figure 19a and the vertical wave velocity magnitude, figure 19c, reveal strong spatial variability whereas the cross-shore wave velocity amplitude, figure 19b is less affected in both $\mathrm{x}$ - and $\mathrm{z}$-directions. The more spatially homogeneous velocity field along the stoss face of the ripple suggests a much less established flow detachment induced ripple vortex generation. Figures 19d and 19e represent colour plots of the time-averaged sand concentration and crossshore sand flux, respectively, along the ripple profile. In these figures, the white solid line represents the position of the lower suspension layer as the height of $8 \%$ volume concentration. It appears clearly that on the lee face of the sand ripple where the velocity field is less homogeneous in the previous figures, the suspension interface reaches higher levels as a consequence of intense local particle entrainment into suspension. Finally, the colour plot in figure $19 \mathrm{e}$, reveals that the strong blue offshore oriented sand flux in the suspension layer appears to originate from the region of strong velocity variability on the lee face of the ripple. This spatial organisation of the mean cross-shore sand flux is in good agreement with the detailed ripple vortex study of Hurther and Thorne (2011).

\subsubsection{Cross-shore sand transport dynamics}


1 In Figures 20a and 20b the intra-wave free-stream wave velocity and the cross-shore vertically integrated transport rates are shown respectively. The total sand transport rate is separated into the bedload and suspended load contributions by vertically integrating the total flux shown in Figure $18 \mathrm{~d}$ over the corresponding vertical region at each relative time $\mathrm{t} / \mathrm{T}$. It can be seen in Figure $20 \mathrm{~b}$ that the bedload transport is smaller in magnitude than the suspended transport rate with a strongly onshore skewed intra-wave dynamics in close agreement with the onshore skewed free-stream velocity. This supports the dominance of the wave-driven component for the bedload transport with negligible sand transport phase lagging effects (Ribberink et al, 2008). The residual net bedload transport is onshore directed with a small value of $0.03 \mathrm{kgm}^{-}$ ${ }^{1} \mathrm{~S}^{-1}$ compared to the total net offshore directed transport of $-0.15 \mathrm{kgm}^{-1} \mathrm{~s}^{-1}$. The intra-wave suspended transport rate is larger in magnitude during both half-cycles with the presence of a strong temporally coherent variation, previously noted during the wave trough, which may be induced by the ejection into the suspension layer of the phase coherent ripple vortex, generated during the wave crest half-cycle on the lee face of the ripple. Offshore transport during the wave trough is seen to dominate leading to a much larger net suspension transport of -0.18 $\mathrm{kgm}^{-1} \mathrm{~s}^{-1}$ compared to the small net onshore bedload transport. The intra-wave dynamics of the suspension transport does not appear to coincide with the free-stream wave velocity in terms of skewness suggesting the presence of sand transport phase lag effects. The coherent structures in the offshore transport may be an indicator of vortex processes and entrainment as described in Hurther and Thorne (2011). The combine suspended load and bedload yield a net total offshore transport of $-0.15 \mathrm{kgm}^{-1} \mathrm{~s}^{-1}$.

Using the co-located and simultaneous high-resolution measurement performance of the ACVP technology, the total net sand transport rate profile can be decomposed into the net current, wave and turbulence driven components. This unique measurement ability allows identification of the relative importance of the different transport processes within the near-bed boundary layer composed of the dense bedload and the dilute suspension layers. The following decomposition of the cross-shore sand flux is applied:

$$
\overline{\mathrm{uC}}\left(z / k_{s}\right)=\overline{\mathrm{u}} \overline{\mathrm{C}}\left(z / k_{s}\right)+\overline{\mathrm{u}_{\mathrm{w}} \mathrm{C}_{\mathrm{w}}}\left(z / k_{s}\right)+\overline{\mathrm{u}^{\prime} \mathrm{c}^{\prime}}\left(z / k_{s}\right)
$$


$1 \quad$ where $\mathrm{u}_{\mathrm{w}}=\left\langle\mathrm{u}(\mathrm{t} / \mathrm{T})>-\overline{\mathrm{u}(\mathrm{t})}, \mathrm{C}_{\mathrm{w}}=\left\langle\mathrm{C}(\mathrm{t} / \mathrm{T})>-\overline{\mathrm{C}(\mathrm{t})}, \mathrm{u}^{\prime}=\mathrm{u}(\mathrm{t} / \mathrm{T})-\mathrm{u}_{\mathrm{w}}-\overline{\mathrm{u}(\mathrm{t})}\right.\right.$ and $\mathrm{c}^{\prime}=\mathrm{C}(\mathrm{t} / \mathrm{T})-\mathrm{C}_{\mathrm{w}}-\overline{\mathrm{C}(\mathrm{t})} \cdot \mathrm{u}(\mathrm{t})$

2 is the cross-shore velocity, $\mathrm{C}(\mathrm{t})$ sediment concentration and $\langle>$ and the overbar respectively

3 represents ensemble wave phased averaged and the temporal mean parameters over 144 wave

4 cycles, respectively. In equation 8 the terms on the right hand side corresponds to current,

5 wave and turbulent components respectively. Figure 21 represents the vertical profiles of all

6 the terms in Equation 8.

7

8 It can be seen that the net onshore bedload transport for $\mathrm{z} / \mathrm{k}_{\mathrm{s}}<0.18$, results from the marginally 9 stronger onshore wave-driven component compared to the net offshore directed current-driven component. The net turbulence-driven flux remains negligibly low compared to the two other components except in the lower bedload layer where it contributes to the net onshore bedload transport in the direction of the leading wave-driven component. In the suspension layer, for $\mathrm{z} / \mathrm{k}_{\mathrm{s}}>0.18$, both the current- and wave-driven components are offshore directed with a negligible turbulence-driven transport. As previously seen in Figure 20, the fact that the wavedriven sand flux does not follow the net onshore directed wave momentum flux (imposed by the positively skewed wave velocity) strongly suggests the existence of suspended ripple vortex entrainment under the form of a phase-coherent convective sand transport phase-lagging effect as previously observed by van der Werf et al (2007) and described in Ribberink et al (2008). The total flux, given by the sum of the three components in equation (8), is seen to be predominantly offshore in the suspended component of the flux but onshore in the near-bed layer, thereby clearly showing the opposite direction of sediment transport for the suspended and nearbed layers.

\subsubsection{Suspended sand transport processes}

The local suspended sand transport processes are discussed in the present section on the basis of the direct measurement of the vertical sand flux dynamics as previously addressed by Smyth et al (2002) in field conditions. Consequently, the vertical sand diffusivity profile is separated into its turbulent and convective components and is analysed in regard to the shape of the mean suspended sediment concentration profile.

Following previous approaches (Nielsen, 1979; Sheng and Hay, 1995; Thorne et al, 2009), in 
1 wave-driven particle flux, $\mathrm{q}_{\mathrm{vw}}$, the turbulent particle flux, $\mathrm{q}_{\mathrm{vt}}$, and the mean depositional settling

2 flux, $\mathrm{q}_{\mathrm{vd}}$, measured as:

6

$$
\varepsilon_{\mathrm{t}}=\overline{\mathrm{w}^{\prime} \mathrm{c}^{\prime}} /(\mathrm{d} \overline{\mathrm{C}(\mathrm{t})} / \mathrm{dz})
$$


7 In Figure 22c the $\mathrm{x}$-axis for the diffusivities have been expressed as a normalised parameter, $8 \varepsilon /\left(U_{o} k_{s}\right)$, following Thorne et. al., 2009. $U_{o}=\sqrt{u_{w}^{2} / 2}$ is the free-stream wave orbital velocity amplitude. It is seen in figure 22c that the magnitude of the normalised wave diffusivity is greater by about a factor of three than the turbulent diffusivity. Further the magnitude of the combined turbulent and wave diffusivity is very comparable to the sediment diffusivity derived for the depositional diffusivity and both are nominally constant with height above the bed in the suspension layer $(\mathrm{z} / \mathrm{ks}>0.18)$. The results in figure $22 \mathrm{c}$ clearly shows the balance between the deposition of particles towards the bed due to gravitational forces and the erosion of particles away from the bed due to turbulent and phase coherent flow motions due to the waveto-wave repeatability of the wave and ripple vortex velocity fields. The exponential form for the concentration profile in figure $22 \mathrm{~b}$ can theoretically be explained by the nominally constant diffusivity with height above the bed, which for the erosive components is dominated by the convective wave diffusivity rather then turbulent diffusivity. The dashed line in figure $22 \mathrm{c}$ is from Van Rijn (1993) and compares favourably with the present observations and further supports the ability of the novel ACVP technology to measure directly the convective and turbulent diffusivity components. For the results presented in figures 17 to 22 normalised parameters were used which required bedform measurements, these were provided by the 3DARP, although they could also have been obtained from the BASSI observations. This again reflects on the complementarity of the acoustic instrument setup used in the Hydralab wave flume studies at Universitat Politècnica de Catalunya (UPC). 


\section{4. Discussion and conclusions}

2 There is an ongoing requirement for instrument development to study near-bed boundary layer sediment transport processes. The complexity of the dynamics requires measurements over a wide range of temporal and spatial scales. Conventional instrumentation has typically made observations at a single height above the bed, frequently without knowledge of the bed morphology. Over the past three decades acoustic systems have been increasingly developed to study boundary layer processes. The principle advantage of acoustics is that profiles of the bedforms, velocity and sediment mobility can be collected with systems which themselves can be mounted unobtrusively away from the near-bed processes under study, yet probe them downwards into the bed itself. Further, it is possible to obtain simultaneous collocated measurement of bedforms, flow and sediment mobility, allowing the dynamic interactions between these three components to be directly analysed. Owing to these capabilities, acoustic developments are ongoing and the purpose of the present work has been to report on the performance of three contemporary research instruments deployed in a complementary way in a large-scale wave flume Hydralab facility.

To measure the micro-topography of the bedforms in the Barcelona wave flume a 3D-ARP was deployed, this provides a series of horizontal transects over a selected swath and radially covering $180^{\circ}$, thereby allowing three dimensional time varying measurements of the surface relief to be obtained. Measurements were collected under clear water and heavy suspended loads, of the order of several $\mathrm{kgm}^{-3}$ and transects of the bed morphology were readily obtained. Unexpectedly the bedforms were not two dimensional as anticipated, but strongly three dimensional, this was clearly evident with the 3D-ARP; however, this would not have been readily identified with a $2 \mathrm{D}$-ARP which provides only a single transect view. Experiments were repeated a number of times at three different wave heights and these have been used to assess the consistency of the measured bedforms. As shown in figures 5a-5d, repeatable values for ripple height, wavelength and slope were obtained with an indicative accuracy at the millimetre scale. Comparison of the ripple dimensions with two contemporary predictors showed $\eta$ and $\lambda$ comparable with the observations, though with ripple slopes approximately half that calculated, which possibly may have been due to the three dimensional nature of the bedforms associated with the use of a medium sized sand rather than coarse sand. As previously observed by O'Donoghue et al (2006) and Hurther and Thorne (2011) such lower inertia particles might even be put into motion by small, low energy, three-dimensional turbulent flow eddies. 
2 The BASSI was developed to study the interactions between the hydrodynamic, bedforms and sediment mobility, with a particular focus on bed features with decimetric wavelengths and centimetric heights, formed under the action of surface waves and/or currents. To assess the internal consistency of the suspended sediment concentrations obtained with the system, time series and averaged vertical profiles were analysed at different frequencies and over the three arrays which composed the BASSI. The results shown in figures 7-9 illustrate the consistency of the results and support the veracity of the BASSI to measure a consistent suspension field across the transect measured by the arrays. To illustrate the time varying 2DHV capability of the BASSI a series of images of the bed and suspended field were presented in figure 11, where it can be clearly seen that suspended sediment structures could be tracked across adjacent images as a wave passed over the arrays. Using the BASSI in a more conventional ABS time averaged mode, measured suspended concentration profiles were compared with two $\mathrm{co}^{\mathrm{mm}}$ only used formulations and reasonable agreement was obtained. Utilising the bed echo from each of the BASSI's 45 transducers a transect of the bed profile was constructed. This yielded bedforms which were consistent over time as shown in figure $13 \mathrm{~b}$ and $13 \mathrm{c}$. The ripple dimension were consistent over different experimental runs for three different wave heights and had comparable dimensions to those obtained from the 3D-ARP.

The ACVP near-bed profiling of 1DV two component velocity, sand concentration, sand flux and bed level tracking was applied to look into detailed near-bed boundary layer dynamics, in the presence of onshore migrating sand ripples, driven by the strongly skewed surface waves in the shoaling zone. As previously observed by Hurther and Thorne (2011), the onshore directed bedload sand transport was in agreement with the onshore ripple migration estimated from the BASSI. The total net sand transport appears to be dominated by the net offshore directed suspended sand transport. Spatial inhomogeneity of the velocity field along a ripple profile suggests that this net offshore suspension transport resulted from the sand entrainment into suspension by the ripple vortex, generated on the lee onshore face of the ripple, after the wave crest-to-trough flow reversal. As shown in figures 21 and 22, the direct measurement of the vertical sand flux and its partition into settling, wave, and turbulent components allowed for direct estimates of the total sand diffusivity decomposed into turbulent and convective contributions. It was found that a vertically constant total sand diffusivity profile prevailed over the entire suspension layer, with a dominant convective diffusivity and a weaker turbulent sand diffusivity. The validity of the constant sand diffusivity in the suspension layer was strongly 
1 supported by the independent measurement of an exponentially decaying mean suspended sand concentration profile. These advanced acoustic measurement performances, recently validated under sheet flow regimes (Fromant et al, 2018), have recently offered new perspectives on near-bed boundary layer sediment transport physics (Naqshband et al, 2014b, 2017; RevilBaudard et al, 2015, 2016; van der Zanden 2016, 2017), in particular for the validation of novel high-resolution simulations obtained with process based two-phase flow-particle models (Cheng et al, 2018).

As mentioned previously the development of instruments to measure sediment transport processes is ongoing and a look at further analysis and advancement of the acoustics instruments reported in the present study is considered. The 3D-ARP measurements described here were generally collected at the end of an experimental run to obtain ripple dimensions, however, with more regular updates of the bedform morphology, it should be possible to estimate bedform migration rates and bedload transport (Lichtman et. al. 2016). Further, the suspended component of the backscattered signal may prove a useful area of investigation. The 3D-ARP used in the present study required around 12 minutes to obtain a maximum resolution scan, however, as systems develop the scan time will almost certainly reduce and using phased array split beam technology could be the next significant step. For the BASSI the focus has been on assessing its capability to obtain internally consistent suspended sediment concentrations across the array and measure bedforms. Given the multi-frequency capability of the BASSI the next step would to obtain 2DHV measurements of the suspended particle size as has been done with ABS in 1DV. Further, given the 2DHV ability, it may be possible to use cross-correlation of the backscattered signal between transducers and PIV type approaches on consecutive images of the suspended sediments to provide information on the flow field, (van Unen et al 1998, Best et al 2010).

Future ACVP perspectives in high-resolution acoustic measurements lie in multi-frequency two-phase acoustic measurements for simultaneous time-resolved measurement of both the fluid flow and particle velocity profiles. A first successful attempt was recently tested by Wilson and Hay (2015b) in dilute particle suspension mixtures using a statistical regularized inversion method. Combined with the present ACVP measurement ability of particle concentration profiling both in the dense bedload and dilute suspension layers, this novel twophase acoustic measurement method would represent a breakthrough for sediment transport research. 


\section{Acknowledgements}

3 The technical support provided by UPC for this instrument flume study is appreciated. Dr Paul

4 Bell of the National Oceanography Centre, UK, is thanked for his software development for 5 interpretation of the 3D-ARP data. The study was supported by the EU Joint Research 6 Activities WISE and COMPLEX respectively part of HydralabIV, contract number 261520

7 and Hydralab+ contract number 261520 and NOC-NERC, UK, Marine Physics and Ocean 8 Climate National Capability funds, and by the French DGA funded ANR-ASMA project 9 MESURE (no. ANR-16-ASMA-0005-01). The authors thank the reviewers for their 10 constructive contribution. 


\section{References}

2 Agrawal Y. C., Whitmire A, Mikkelsen Ole A. and Pottsmith H. C. 2008. Light scattering by 3 random shaped particles and consequences on measuring suspended sediments by laser 4 diffraction. Journal Geophysical Research, 113, C04023; 1-11, doi:10.1029/2007JC004403.

6 Agrawal, Y. C., and D. M. Hanes. 2015. The implications of laser-diffraction measurements of 7 sediment size distributions in a river to the potential use of acoustic backscatter for sediment measurements, Water Resources. Research., 51, doi:10.1002/2015WR017268.

Amoudry, L.O. And Souza, A.J., 2011. Deterministic Coastal Morphological and Sediment Transport Modeling: A Review and Discussion. Reviews of Geophysics, 49, RG2002.

Baumert, H., Chapalain, G., Smaoui, H., McManus, J.P., Yagi, H., Regener, M., Sundermann, J., and Szilagy, B., 2000. Modelling and numerical simulation of turbulence, waves and suspended sediments for pre-operational use in coastal seas. Coastal Engineering, 41, 63-93.

Bell, P.S., Thorne, P.D., 1997a. Application of a high resolution acoustic scanning system for imaging sea bed microtopography. Seventh International Conference on Electronic Engineering in Oceanography. Held at the Southampton Oceanographic centre 23-25 June 1997. IEE Conference Publication No. 435, 128-133.

Bell P. S. and Thorne P. D. 1997b. Measurement of sea bed evolution in an estuarine environment using a high resolution acoustic sand ripple profiling system. MTS/IEEE Conference Proceedings Oceans '97. Held 6-9 October in Halifax Canada. DOI: 10.1109/OCEANS.1997.634386. 339-343.

Bell, P.S., Thorne, P.D., Williams, J.J., 1998. Acoustic measurements of sand ripple profile evolution under controlled wave conditions. Proceedings of the fourth European Conference on Underwater Acoustics, held in Rome, Sept 21-25 1998 Vol.1, 353-358.

Bell P. S. and Thorne P. D. 2007. Field measurements of wave induced sand ripples in three dimensions. Published in the 'Underwater Acoustic Measurements: Technologies and results' conference CDrom, held on Crete 25-29 June at F.O.R.T.H. pp 1045-1052. 
2 Best J., Simmons S., Parsons D., Oberg K., Czuba J. and Malzone C. 2010. A new methodology for quantitative visualisation of coherent flow structures in alluvial channels using multibeam echo-sounding (MBES). Geophysical Research Letters, vol 37, L06405. 1-6

6 Betteridge, K.F.E., Thorne, P.D. and Cooke, R.D., 2008. Calibrating multi-frequency acoustic 7 backscatter systems for studying near-bed suspended sediment transport processes. Continental 8 Shelf Research., 28, 227-235.

Bunt J. A. C., Larcombe P. and Jago C. F. 1999. Quantifying the response of optical backscatter devices and transmissometers to variations in suspended particulate matter. Continental Shelf research, 19, 1199-1220.

Cellino M., and Graf W.H. 1999. Quantifying the response of optical backscatter devices and transmissometers to variations in suspended particulate matter. Journal of Hydraulic Engineering, 125(5), 455.

Cheng, Z., T.-J. Hsu, and J. Chauchat (2018). An eulerian two-phase model for steady sheet flow using large-eddy simulation methodology. Advances in Water Resources, 111(Supplement C):205-223.

Crawford, A.M., Hay, A.E., 1993. Determining suspended sand size and concentration from multifrequency acoustic backscatter. J. Acoust. Soc. Am. 94(6), 3312-3324.

Davies, A.G., van Rijn, L.C., Damgaard, J.S., van de Graaff, J., and Ribberink, J.S., 2002. Intercomparison of research and practical sand transport models, Coast. Eng., 46, 1-23.

28 Davies A. G. and Thorne P.D. 2008. Advances in the study of moving sediments and evolving seabeds. Surveys in Geophysics, Vol 29, No 1, January, 1-36.

Davies A. G. and, Thorne P.D. 2016. On the suspension of graded sediment by waves above ripples: Inferences of convective and diffusive processes. Continental Shelf Research 112 4667. 
1 Dolphin T and Vincent C. 2009, The influence of bed forms on reference concentration and suspension under waves and currents. Continental Shelf Research, 29,412-432.

Downing, A., Thorne, P.D. and Vincent, C.E., 1995. Backscattering from a suspension in the near field of a piston transducer. Journal Acoustical Society of America, 97 (3), 1614-1620.

Dyer, K.R. and Soulsby R.L., 1988. Sand transport on the continental shelf. Annual Review 8 Fluid Mechanics, 20, 295-324.

Fromant G., Myeras R., Revil-Baudard T., Puleo J., Hurther D. and Chauchat J. 2018. On bedload and suspended load measurement performances in sheet flows using acoustic (ACVP) and conductivity (CCP) profilers. Journal of Geophysical Research, Earth Surface, under 13 review.

Goldstein E. B., Coco G and Murray A.B. 2013. Prediction of wave ripple characteristics using genetic programming. Continental Shelf Research, 71, 1-15.

Graham G.W., Davies E.J., Nimmo-Smith W. A. M., Bowers D. G., and Braithwaite K. M. 2012. Interpreting LISST-100X measurements of particles with complex shape using digital in-line holography. Journal of Geophysical Research, 117, C05034, 1-20, doi:1029/2011JC007613.

Hardisty J. 1990. Beaches form and processes. Published by Unwin Hyman Ltd, London. pp324.

Hay, A.E., 1991. Sound scattering from a particle-laden turbulent jet, J. Acoust. Soc. Am., 90, 2055-2074.

Hay, A.E., Wilson, D., 1994. Rotary sidescan images of nearshore bedform evolution during a storm. Marine Geo. 119, 57-65.

Hay, A.E., and Bowen, A.J., 1994. Coherence scales of wave-induced suspended sand concentration fluctuations. Journal of Geophysical Research 99 (C6), 12749-12765. doi:10.1029/94JC00290. 
2 Hay A. E., 2011. Geometric bottom roughness and the bed state storm cycle. Journal of

3 Geophysical Research, 116. C04017, doi:1029/2010JC006687.

5 Hay A.E., Zedel L., Cheel R. And Dillon J., 2012. On the vertical and temporal structure of 6 flow and stress within the turbulent oscillatory boundary layer above evolving beds. 7 Continental Shelf Research, 46, 31-49.

8

9 Heathershaw, A.D. and Thorne P.D, 1985. Sea-bed noises reveal role of turbulent bursting 10 phenomenon in sediment transport by tidal currents. Nature, 316, 339-342.

Hess, F.R. and Bedford, K.W., 1985. Acoustic Backscatter System (ABSS): the instrument and some preliminary results. Mar. Geol., 66, 357-379.

Hurther D. and Lemmin U., 1998. A constant-beam-width transducer for 3D acoustic Doppler profile measurements in open-channel flow. Meas. Sci. Technol. 9(10), 1706-1714.

Hurther D. and Lemmin U., 2000. Shear stress statistics and wall similarity analysis in turbulent boundary layers using a high resolution 3D ADVP. IEEE J. of Oceanic Engineering 25(4): 446457.

Hurther D. and Lemmin U., 2001. A correction method for turbulence measurements with a 3D acoustic Doppler velocity profiler. J. Atmos. Oceanic Technol. 18(3), 446-458.

Hurther D. and Lemmin U, 2008. Improved turbulence profiling with field adapted Acoustic Doppler Velocimeters using a bi-frequency Doppler noise suppression method. J. of Atmos. and Oceanic Technol. 25 (2), 452-463.

Hurther D. and Lemmin U., 2003. Turbulent particle and momentum flux statistics in suspension flow. Water Resources Research, 39, 1139, doi:10.1029/2001WR001113, 5.

Hurther D., Lemmin U., Terray E. A., 2007. Turbulent transport in the outer region of rough wall open-channel flows: the contribution of Large Coherent Shear Stress Structures (LC3S). Journal of Fluid Mechanics 574, 465-493. 
2 Hurther, D., Thorne, P.D., Bricault, M., Lemmin, U., and Barnoud, J.M., 2011. A multi3 frequency Acoustic Concentration and Velocity Profiler (ACVP) for boundary layer 4 measurements of fine-scale flow and sediment transport processes. Coastal Engineering, 58 5 (7), 594-605.

6

7 Hurther D. and Thorne P.D. 2011. Suspension and near-bed load sediment transport processes 8 above a migrating, sand-rippled bed under shoaling waves. J. Geophys. Res., Vol 116, C07001, 9 doi:1029/2010JC006774. NEW347007

James, I.D., 2002. Modelling pollution dispersion, the ecosystem and water quality in coastal waters: a review. Environmental Modelling and Software, 17 (4), 363-385.

Kineke, G.C. and Sternberg, R.W., 1992. Measurements of high concentration suspended sediments using the optical backscatterance sensor. Mar. Geol., 108: 253-258.

Kramer K and Winter C. 2016. Predicted ripple dimensions in relation to precision of in situ measurements in the southern North Sea. Ocean Sciences, 12,1221-1235. doi:10.5194/os-12$1221-2016$

Larsen S. M., Greenwood B. and Aagaard T. 2015. Observations of megaripples in the surf zone. Marine Geology 364, 1-11.

Lemmin, U. and Rolland, T. 1997. Acoustic Velocity Profiler for Laboratory and Field Studies. J. Hydr. Engr., 123(12).

Lee, G., Dade, W.B., Friedrichs, C.T., and Vincent, C.E., 2004. Examination of reference concentration under waves and currents on the inner shelf. J. Geophys. Res. 109. C02021, doi:10.1029/2002JC001707.

Lee, C., Wu, C.H., and Hoopes, J.A., 2009. Simultaneous particle size and concentration measurements using a back-lighted particle imaging system. Flow Measurement and Instrumentation, 20 (4-5), 189-199. 
1 Lhermitte R. and Lemmin U., 1994. Open-channel flow and turbulence measurements by high-

2 resolution Doppler Sonar. J. Atmos. Oceanic Technol., 11(5), 1295-1308.

4 Lichtman, Ian D, Thorne Peter D, Baas Jaco H and Amoudry Laurent O. 2016. Bedform 5 migration in an intertidal environment influenced by cohesion, pp 119-122. Van Landeghem, 6 K.J.J., Garlan T. and Baas, J.H. (Eds), 2016. MARID 2016. Fifth International. Conference on 7 Marine and River Dune Dynamics, Caernarfon, United Kingdom, 4-6 April 2016. Bangor 8 University and SHOM. 216 pp. ISBN 978-2-11-128417-3.

Liu P and Lam K. M. 2015. Simultaneous PIV measurements of fluid and particle velocity fields of a sediment-laden buoyant jet. Journal of Hydro-environment Research, 9, 314-323. Lu Y., Li S., Zuo L. Liu H. and Roelvink J. A. 2015. Advances in sediment transport under combined action of waves and currents. International Journal of Sediment Research, 30, 351360.

Malarkey J, Magar V. and Davies A.G. 2015. Mixing efficiency of sediment and momentum above rippled beds under oscillatory waves. Continental Shelf Research, 108, 76-88.

Moate BD, Thorne PD and Cooke RD. 2011. Acoustic Backscatter measurements in two dimensions. The acoustic suspended sediment imager. Proceedings of the 4th International conference on Underwater Acoustic Measurements:Technologies and Results, Kos, Greece. Edited by John S. Papadakis and Leif Bjorno. 1579-1584.

Moate B.D. and Thorne P.D. 2012. Interpreting acoustic backscatter from suspended sediments of different and mixed mineralogical composition. Continental Shelf Research, 46, 67-82.

Moate B. D., Thorne P. D. and Cooke R. D. 2016. Field deployment and evaluation of a prototype autonomous two dimensional acoustic backscatter instrument: The Bedform And Suspended sediment Imager (BASSI). Continental Shelf Research 112 78-91

Naqshband S., Ribberink J. S., Hurther D., Barraud P. A. and Hulscher S. J.M.H. 2014a. Experimental evidence for turbulent sediment flux constituting a large proportion of the total sediment flux along migrating sand dunes. Geophysical Research Letters, 41, doi:10.1002/2014GL062322, 1-9. 
2 Naqshband S, Ribberink, J. S, Hurther D., Barraud, P.A., and Hulscher, S.J.M.H. 2014b.

3 Experimental evidence for turbulent sediment flux constituting a large portion of total sediment

4 flux along migrating sand dunes. Geophysical Research Letters. doi: 10.1002/2014GL062322. 5

6 Naqshband S, Hoitink, A.J.F., McElroy B., Hurther D., and Hulscher, S.J.M.H. 2017. A sharp

7 view on river dune transition to upper stage plane bed. Geophysical Research Letters. In press 8

9 Nielsen P. 1979. Some basic concepts of wave sediment transport. IHHE Series Paper 20, Technical University of Denmark, 160 pp.

Nielsen P. 1992. Coastal Bottom Boundary Layers and Sediment Transport. Advanced series on ocean engineering, volume 4. World Scientific, Singapore, $324 \mathrm{pp}$.

O'Donoghue T, Doucette J S, van der Werf J J and Ribberink J S. 2006. The dimensions of sand ripples in full-scale oscillatory flows. Coastal Engineering 53 (2006) 997-1012.

O'Hara Murray, R. B., Thorne, PD and Hodgson DM. 2011. Intrawave observations of sediment entrainment processes above sand ripples under irregular waves, J. Geophys. Res., 116, C01001, doi:10.1029/2010JC006216.

O’ Hara Murray, R. B., Hodgson D.M. and Thorne P.D. 2012. Wave groups and the character of sediment resuspension over an evolving sandy bedforms. Continental Shelf Research, 46, 16-30.

Puleo J. A., Johnson R. V., Butt T., Kooney T. N. and Holland K. T. 2006. The effect of bubbles on optical backscatter sensors. Marine Geology, 230,87-97.

Rai, A. K., and Kumar, A. (2015). Continuous measurement of suspended sediment concentration: Technological advancement and future outlook. Measurement. 76, 209-227. on boundary layer mixing and mass flux across the sediment-water interface. Water Resourc. Res., 46, W07530, doi:10.1029/2009WR008248. 
1 Revil-Baudard, T., Chauchat, J., Hurther, D. and Barraud, P.-A. 2015. Investigation of sheet-

2 flow processes based on novel acoustic high-resolution velocity and concentration 3 measurements. Journal of Fluid Mechanics. 767, 1-30.

4

5 Revil-Baudard, T., Chauchat, J., Hurther, D. and Eiff, O. 2016. Turbulence modifications 6 induced by the bed mobility in intense sediment laden flows. Journal of Fluid Mechanics. 808, $7 \quad 469-484$.

8

9 Ribberink, J.S., van Der Werf, J.J., O’Donoghue, T., and Hassan, W.N.M, 2008. Sand motion induced by oscillatory flows: Sheet flow and vortex ripples. Journal of Turbulence, 9 (20), 1 32.

Rouse, H. 1937. Nomogram for the settling velocity of spheres. In: Division of Geology and 14 Geography, Exhibit D of the Report of the Commission on Sedimentation, 1936-37, National 15 Research Council, Washington, D.C., pp. 57-64. RUBEY, W.W. 1933. Settling velocities of gravel, sand, and silt.

Schaafsma A. S. and Hay A. E. 1997. Attenuation in suspensions of irregularly shaped sediment particles: A two-parameter equivalent spherical scatterer model. Journal Acoustical. Society of America. 102, 1485-1502.

Shen C and Lemmin U (1999). Application of an acoustic particle flux profiler in particle-laden open-channel flow. J. Hydr. Res., 37(3), 407-419.

Sheng J and Hay A. E. 1995. Sediment eddy diffusivities in the nearshore zone, from multifrequency acoustic backscatter. Continental Shelf Research, 15, 2/3, 129-147.

Smyth, C.E., Zedel, L. and Hay, A.E., 2002. Coherent Doppler profiler measurements of nearbed suspended sediment fluxes and the influence of bedforms. J. Geophys. Res. 107 (C8), 19.130 19.20 . 
1 Soulsby R. L., Whitehouse R. J. S. and Martin K. V. 2012. Prediction of time evolving sand 2 ripples in shelf seas. Continental Shelf Seas, 38, 47-62.

4 Stanton, T. P. 1996. Coherent Acoustic Sediment Flux Probe, U.S. Army Corps of Engineers, 5 Contract Report, CERC-96-1. September 1996.

6

7 Thorne, P.D. and Hardcastle, P.J., 1997. Acoustic measurements of suspended sediments in 8 turbulent currents and comparison with in-situ samples. Journal of the Acoustical Society of 9 America 101 (5) (Pt. 1), 2603-2614.

Thorne, P.D. and Hanes, D.M., 2002. A review of acoustic measurement of small-scale sediment processes. Cont. Shelf Res., 22, 603-632.

Thorne, P.D., Williams, J.J., and Davies, A.G., 2002. Suspended sediments under waves measured in a large scale flume facility. J. Geophys. Res. 107(C8), 4.1-4.16.

Thorne P. D., Davies A.G. and Williams J.J. 2003. Measurements of near-bed intra-wave sediment entrainment above vortex ripples. Geophysical research Letter Vol 30, NO. 20, 2028, 1-4,doi:10.1029/2003GL018427.

Thorne, P.D. and Meral, R., 2008. Formulations for the scattering properties of sandy sediments for use in the application of acoustics to sediment transport. Journal of Continental Shelf Research, 28, 309-317.

Thorne, P. D., Davies A. G and Bell P. S. 2009. Observations and analysis of sediment diffusivity profiles over sandy rippled beds under waves, J. Geophys. Res., 114, C02023, doi:10.1029/2008JC004944.

Thorne P. D., Hurther D. and Moate B. D. 2011. Acoustic inversions for measuring boundary layer suspended sediment processes. J Acoust Soc Amer 130(3) 1188-1200. DOI:10.1121/1.3618728. NEW302013

Thorne P. D. Hurther D, Cooke R.D., Barraud P.A.,Barnoud J.M., Caceres I and SanchesArcilla A. 2013. On the study of boundary layer transport processes using new developmental 
1 acoustic techniques. In the proceeding of Coastal Dynamics 2013, held in Arachon, France, 2 24-28 June. Pp1667-1676.

4 Thorne P.D. and Hurther D. 2014. An Overview on the use of backscattered sound for 5 measuring suspended particle size and concentration profiles in non-cohesive inorganic 6 sediment transport studies. Continental Shelf Research. 73, 97-118.

7

8 Thorne P. D. and Hurther D. 2017. Perspectives of ongoing developments for measuring 9 10 sediment dynamics. Journal Acoustical Society of America. Vol 141, No 5 Pt2 of 2, 36693670 .

Traykovski P., Latter R. J and Irish J. D. 1999a. A laboratory evaluation of the laser in situ scattering and transmissometery instrument using natural sediments. Marine Geology, 159, 355-367.

Traykovski, P., Hay, A.E., Irish, J.D., Lynch, J.F., 1999b. Geometry, migration, and evolution of wave orbital ripples at LEO-15. J. Geophys Res, Vol.104, No.C1, 1505-1524.

Traykovski P., 2007. Observations of wave orbital scale ripples and a nonquilibrium timedependent model. Journal of Geophysical Research, 112, C06026, 19pp, doi:10.1029/2006JC003811.

van der Werf J J, Ribberink, J S, O'Donoghue T and Doucette J S. 2006. Modelling and measurement of sand transport processes over full-scale ripples in oscillatory flow. Coastal Engineering 53 (2006) 657-673.

van der Werf J J, Doucette J S, O’Donoghue T and Ribberink J S. 2007. Detailed measurements of velocities and suspended sand concentrations over full-scale ripples in regular oscillatory flow. Journal of Geophysical Research, vol 112, F02012, doi:10.1029/2006JF000614.

Van der Zanden, J., van der A, D. A., Hurther, D., Caceres, I, O’Donoghue, T. and Ribberink, J. S., 2016, Near-bed hydrodynamics and turbulence below a large-scale plunging breaking 
1 wave over a mobile barred bed profile, J. Geophys. Res. Oceans, 121, 6482-6506, doi:10.1002/2016JC011909.

4 Van der Zanden, J., van der A, D. A., Hurther, D., Caceres, I, O’Donoghue, T. and Ribberink, 5 J. S., 2017, Suspended sediment transport around a large-scale laboratory breaker bar, Coastal 6 Engineering, 125, 51-69, doi: 10.1016/j.coastaleng.2017.03.007.

7

8 Van Rijn, L. C. (1993), Principles of Sediment Transport in Rivers, Estuaries and Coastal Seas, 9

\section{3 pp., Aqua Publ., Amsterdam.}

Van Unen R. F., Thorne P. D., Cox H and Kamminga S. D. 1998. Laboratory measurements of current flow using cross correlation on acoustic backscattering from suspended sediments. 1998. J. Acoust. Soc. Am. 104 (3), Pt. 1, 1345-1355.

Villard P V and Osborne P D. 2002. Visualization of wave-induced suspension patterns over two-dimensional bedforms. Sedimentology (2002) 49, 363-378

Vincent, C.E., Hanes, D.M. and Bowen, A.J., 1991. Acoustic measurements of suspended sand on the shoreface and the control of concentration by bed roughness. Mar. Geo., 96, 1-18.

Wang, B., Liao, Q., Bootsma, H. A. and Wang, P.F. (2012) A Dual-Beam-Dual-Camera method for battery-powered in situ PIV system. Experiments in Fluids. 52(6), 1401-1414.

Wang B., and Liao, Q. (2016) Field observations of turbulent dissipation rate profiles immediately below the air-water interface. Journal of Geophysical Research: Ocean doi: 10.1002/2015JC011512.

Williams J.J, Bell P. S., Thorne P. D. Trouw K., Hardcastle P. J. and Humphery. 2000. Observed and predicted vertical suspended sediment concentration profiles and bedforms in oscillatory-only flow. Journal of Coastal Research, 16(3), 698-708. ISSN 0749-0208

Williams, J.J., Bell, P.S., and Thorne, P.D., 2003. Field measurements of flow fields and sediment transport above mobile bed forms. Journal of Geophysical Research-Oceans, 108, C4, 3109, doi:10.1029/2002JC001336, 6,1-66. 
2 Williams, J.J., Bell, P.S., and Thorne, P.D., 2005. Unifying large and small wave-generated 3 ripple. Journal of Geophysical Research-Oceans, 110, C02008, 1-18, 4 doi:10.1029/2004JC002513.

6 Wilson G. W. and Hay A. E. 2015a. Acoustic backscatter inversion for suspended sediment 7 concentration and size: A new approach using statistical inverse theory. Continental Shelf 8 Research, 106, 130-139.

Wilson, G.W. and Hay, A.E., 2015b. Measuring two-phase particle flux with a multi-frequency acoustic Doppler profiler. Journal of the Acoustical Society of America, 138(3), 3811.

Wilson, G. W., and A. E. Hay. 2016. Acoustic observations of near-bed sediment concentration and flux statistics above migrating sand dunes, Geophys. Res. Lett., 43, 6304-6312, doi:10.1002/2016GL069579.

Yu K. and Xu J. 2016. Adaptive PIV algorithm based on seeding density and velocity information. Flow Measurement and Instrumentation, 51, 21-29.

Zedel, L. Hay, A.E. Cabrera, R. and Lohrmann, A. 1996: Performance of a single beam pulse to pulse coherent Doppler profiler, IEEE J. Ocean. Eng. 21, 290-297.

Zedel, L. and Hay, A.E., 1999. A coherent Doppler profiler for high resolution particle velocimetry in the ocean: laboratory measurements of turbulence and particle flux. J. Atmos.

Oceanic Technol. 16, 1102-1117 


\section{7. Figure Caption}

2

3 Figure 1. a) Photograph of the instrumentation in the UPC wave flume showing the 34 Dimensional Acoustic Ripple Profiler, 3D-ARP, the Bedform And Suspended Sediment 5 Imager, BASSI and the high resolution Acoustic Concentration and Velocity Profilers, 6 ACVP's, with an expanded image in b) of the ACVP's locations.

Figure 2. a) and b) respectively provide plan view and cross-shore schematics of the instrumentation layout. The still water level in the flume was at $1.6 \mathrm{~m}$ above the sand-bed at the location of the instruments.

Figure 3. A single transect swath image of the relative backscattered signal from the 3D-ARP and identification of the bed echo indicated by the white line for; a) at the end of an experiment in clear water and b) during an experiment with sediment in suspension. The colour scale represents the relative backscattered signal level.

Figure 4 a) An image of the bed in the flume from the 3D-ARP for $\mathrm{H}=0.5 \mathrm{~m}$, the vertical structure on the right hand side of the image is a reflection from a side wall. b) Measurements for $\mathrm{H}=0.4 \mathrm{~m}$ of the ripple height, $\eta$, and wavelength, $\lambda$, for transect rotation angles between $\theta=0^{\circ}-180^{\circ}$. c) Photographs of the bed taken at the time of the experiment, the dimensions labelled are approximate.

Figure 5. Measurements from repeated experimental runs at wave heights $\mathrm{H}=0.3 \mathrm{~m}$ (o), $0.4 \mathrm{~m}$ (x) and $0.5 \mathrm{~m}$ ( $\square$ ) for ripple; a) height, b) wavelength, c) slope and d) the difference in heights between adjacent transects normalised by the mean ripple height for the experimental run. The lines represent a mean value for each value of $\mathrm{H}$.

Figure 6. Comparison of two ripple predictors, Soulsby et al 2012, equation (1) and Goldstein et al 2013, equation (2), with normalised ripple dimensions. A is the wave orbital amplitude at the bed and $\mathrm{d}_{50}$ the median diameter of the bed sediments. The symbols refer to the same values of $\mathrm{H}$ as in figure 5. 
2 Figure 7. Time series of the suspended sediment concentration with height above the bed for 3 array SN:001 measured at the three frequencies; a) $0.75 \mathrm{MHz}$, b) $1.25 \mathrm{MHz}$ and c) $2.5 \mathrm{MHz}$. 4 For figures $7-11 \mathrm{H}=0.5 \mathrm{~m}$.

5

6 Figure 8. Time series of the suspended sediment concentration with height above the bed 7 averaged across each array for; a) array SN:001, b) array SN:002 and c) array SN:003.

9 Figure 9. Mean suspended sediment concentration profiles averaged across an array and over 10 the period of the experiment for; a) array SN:001, b) array $\mathrm{SN}: 002$ and c) array SN:003. The 11 solid line with error bars is the average of the three frequencies.

13 Figure 10. Spatial measurement of the quasi-instantaneous suspended sediment field across the $14 \quad 1.5 \mathrm{~m}$ BASSI.

Figure 11. Sequential spatial measurements of the suspended sediment field at $2 \mathrm{~Hz}$ over the $1.5 \mathrm{~m}$ transect during the passage of a wave over the BASSI. The arrows at the top of the panels indicate the direction of flow and the colour bar in figure 10 represents the concentrations in the present figure.

Figure 12. Comparison of an empirical exponential fit, Nielsen 1992, equation (6) and Rouse power law equation (7) with the BASSI spatial and temporal averaged normalised suspended concentration profiles for; a) $\mathrm{H}=0.3 \mathrm{~m}$, b) $\mathrm{H}=0.4 \mathrm{~m}$ and c) $\mathrm{H}=0.5 \mathrm{~m}$.

Figure 13. Measurements of bedforms from the BASSI during an experiment with $\mathrm{H}=0.4 \mathrm{~m}$. a) A single transect image of the backscattered signal and identification of the bed echo indicated by the white line, b) evolution of a transect over time and c) variation of ripple height and wavelength with time and mean values given by the solid lines. 
1 Figure 14. BASSI measurements from repeated experimental runs at wave heights $\mathrm{H}=0.3 \mathrm{~m}$ 2 (o), $0.4 \mathrm{~m}(\mathrm{x})$ and $0.5 \mathrm{~m}(\square)$ for ripple; a) height, b) wavelength and c) slope. The lines represent 3 a mean value for each value of $\mathrm{H}$.

5 Figure 15. Comparison of the BASSI and ACVP3 suspended concentrations with height above 6 the bed for $\mathrm{H}=0.5 \mathrm{~m}$

8 Figure 16. Intra-wave free stream velocity measured at a height above the ripple crest of $\mathrm{z}=0.1$

$9 \mathrm{~m}$ for a wave height of $\mathrm{H}=0.5 \mathrm{~m}$.

11 Figure 17. Profiles of; a) root-mean-square velocity, b) mean current, c) mean normalised sand 12 concentration and d) total sand transport rate in the cross-shore direction. All data correspond 13 to measurements with a wave height $\mathrm{H}=0.5 \mathrm{~m}$.

Figure 18. Intra-wave measurements of; a) free-steam wave velocity, b) wave velocity profiles, c) relative concentration profiles d) sand flux profiles in cross-shore direction, e) relative concentration inside the bedload layer and f) relative concentration in the suspension layers. The black solid line in figures b) and d) represent the interface separating the lower bedload from the upper suspension layer (following the height of the $8 \%$ by volume concentration contour). The white horizontal dashed lines in figure c) represent the selected heights presented in figures e) and f).

Figure 19. ACVP measurements along one ripple profile of (a) time-averaged cross-shore velocity $\mathrm{u}$, (b) cross-shore wave-velocity magnitude $|\mathrm{u}|$, (c) vertical wave-velocity magnitude $|w|,(d)$ base ten logarithm of concentration, $C$ and (e) cross-shore sand flux uC. The $\mathrm{x}$-axis is calculated assuming a constant ripple migration speed measured by the BASSI for a wave height of $0.5 \mathrm{~m}$.

Figure 20. a) Wave free-stream velocity, b) intra-wave sand vertically integrated transport rates measured by ACVP3 for the bedload and suspended load. 
2 Figure 21. Total cross-shore sand transport profile and its decomposition into turbulent, current 3 and wave components as give in Equation 8. The data was measured with ACVP3 for a wave 4 height $0.5 \mathrm{~m}$.

5

6 Figure 22. ACVP3 measured; a) profiles of vertical sand fluxes decomposed into turbulent, 7 wave-driven and depositional particle fluxes, b) time-averaged concentration profile and its 8 exponential fit in the suspension layer $\left(\mathrm{z} / \mathrm{k}_{\mathrm{s}}>0.18\right)$ and $\left.\mathrm{c}\right)$ profiles of normalized sand 9 diffusivities decomposed into turbulent, convective, their (negative) combination and total 10 diffusivity obtained from the depositional flux. The dash line in c) is the model of Van Rijn 11 (1993).

12 

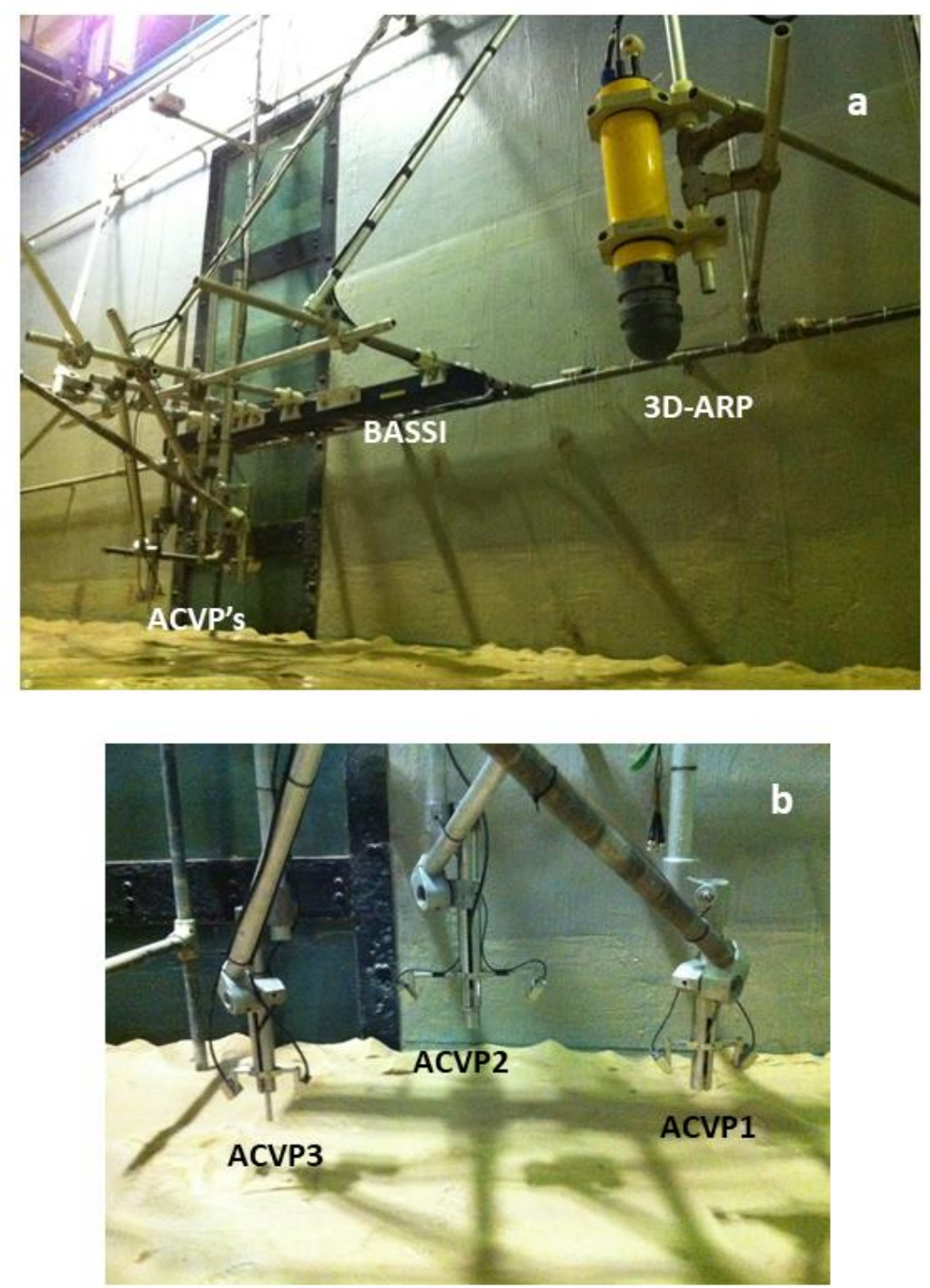

2

$3 \quad$ Fig 1

4 


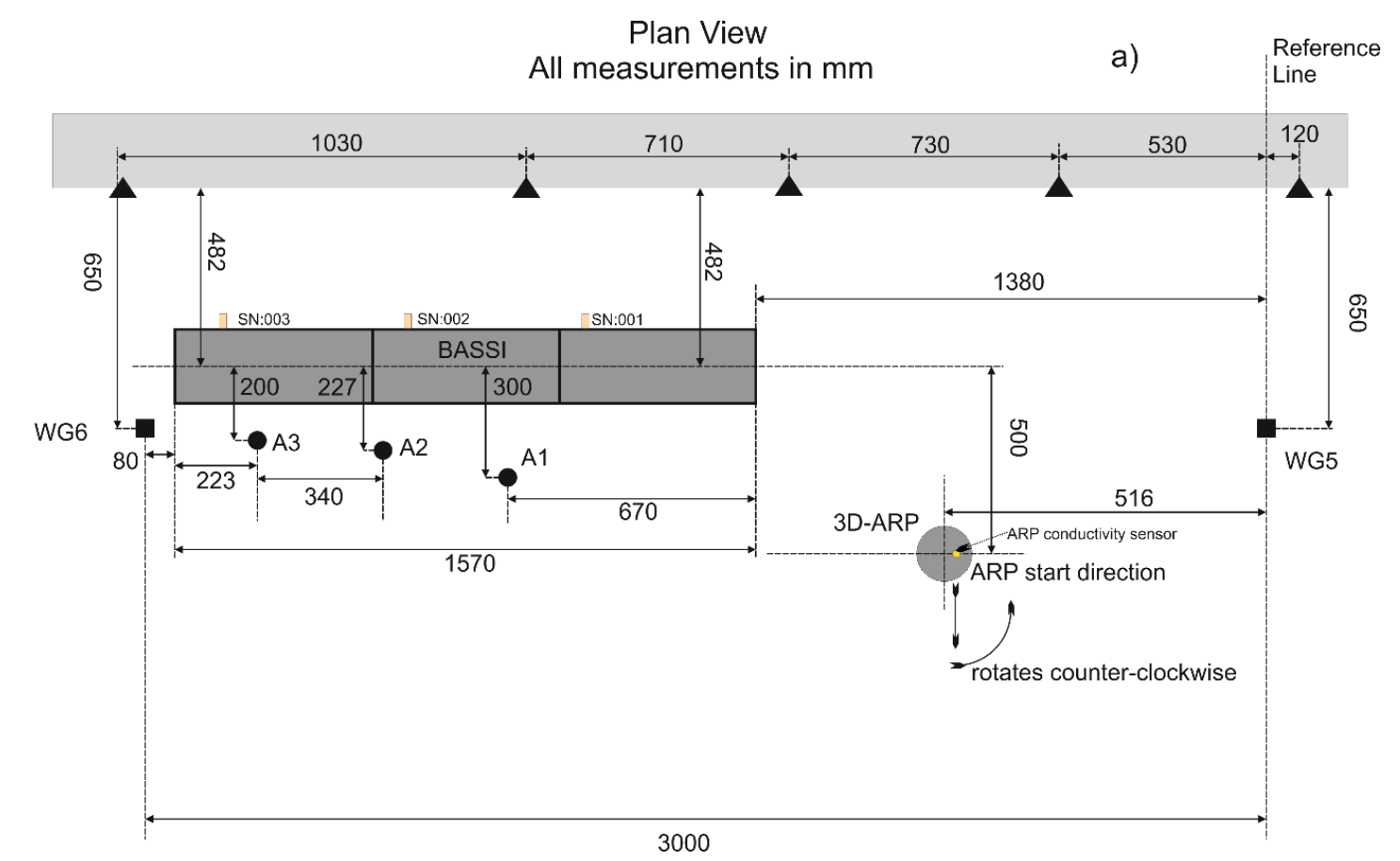

- Triangles are pressure sensors

Squares are wave gauges

1

Circles are ACVP positions

Wave direction

2

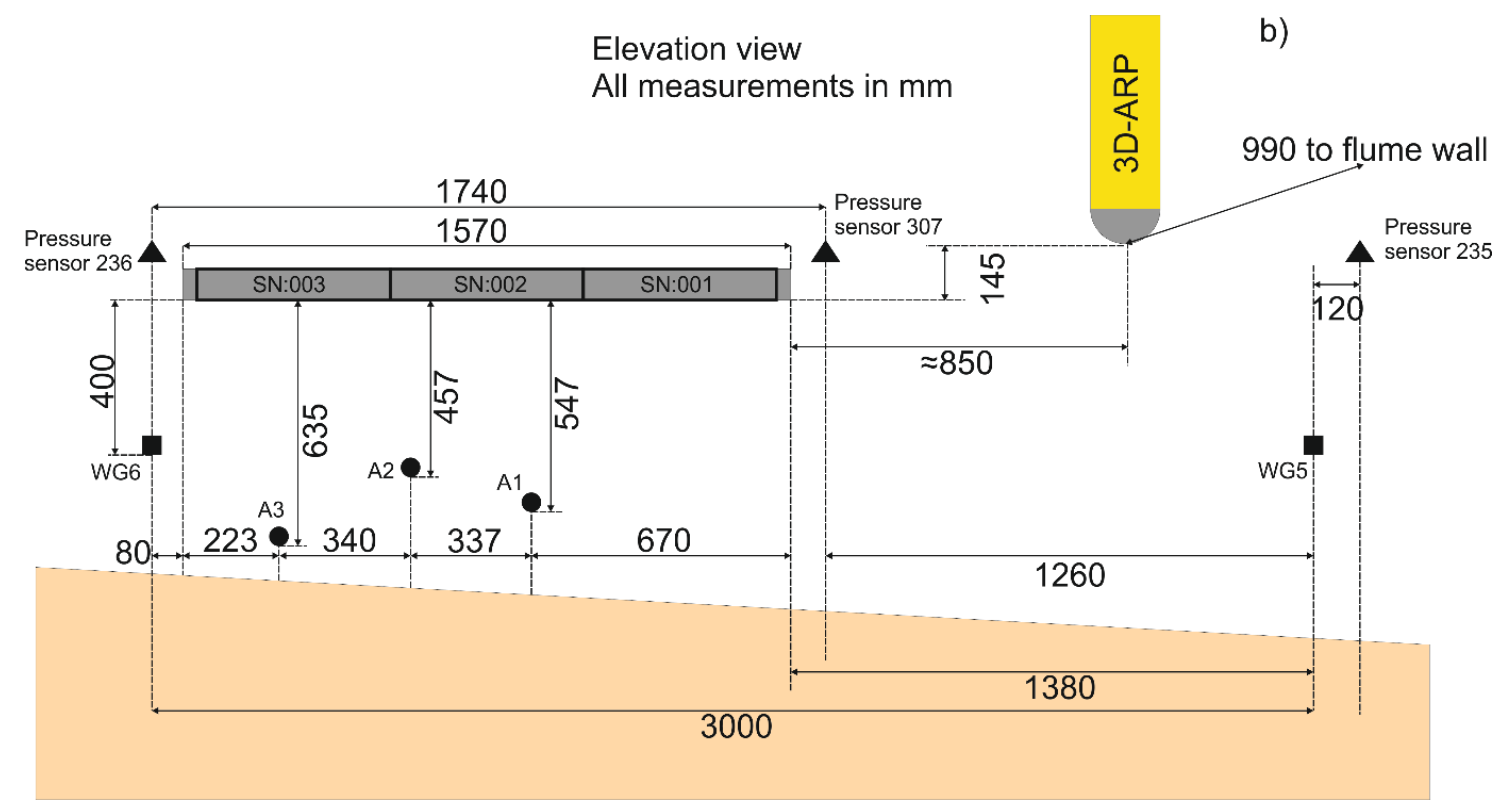

$5 \quad$ Fig 2 

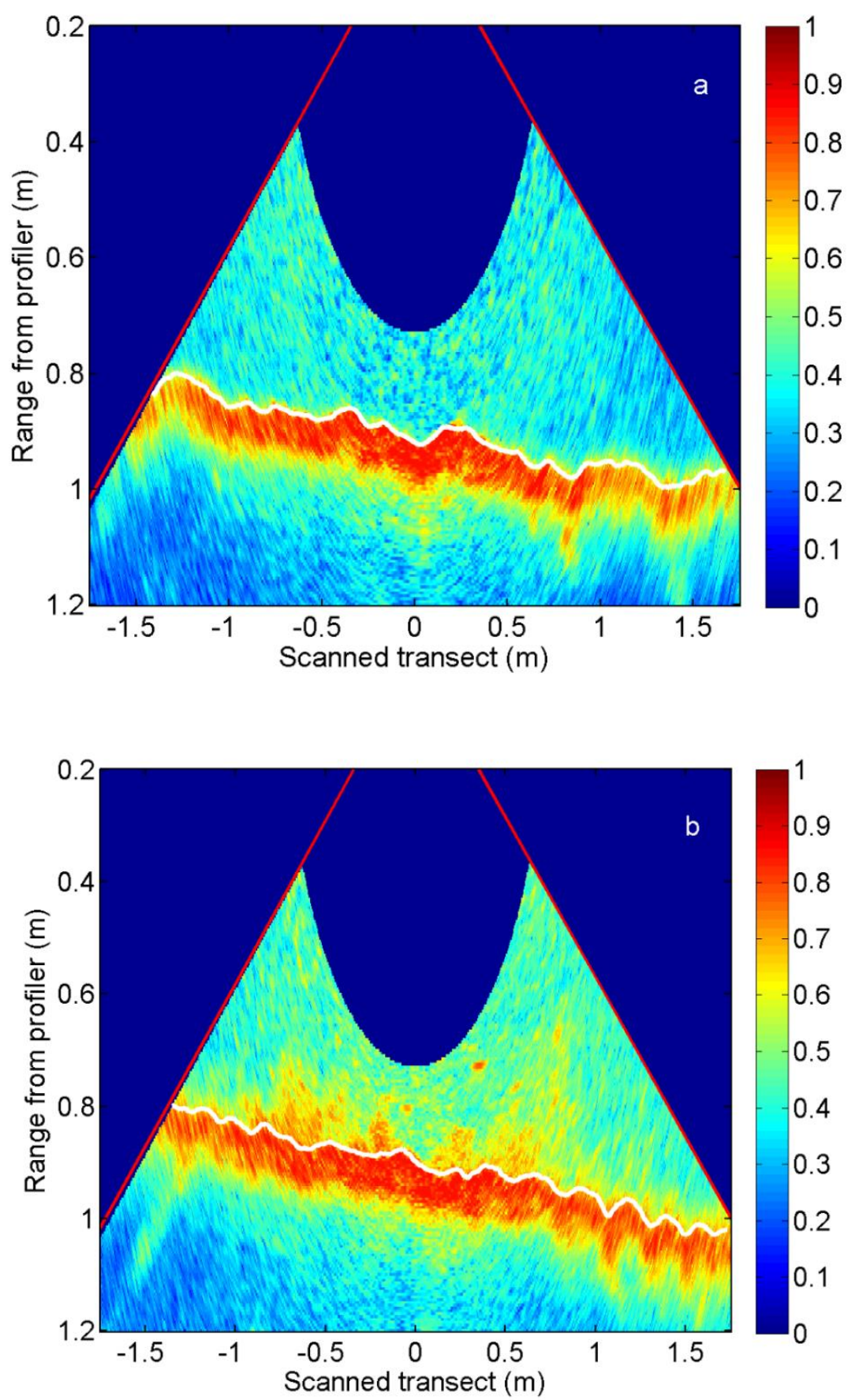

$3 \quad$ Fig 3 


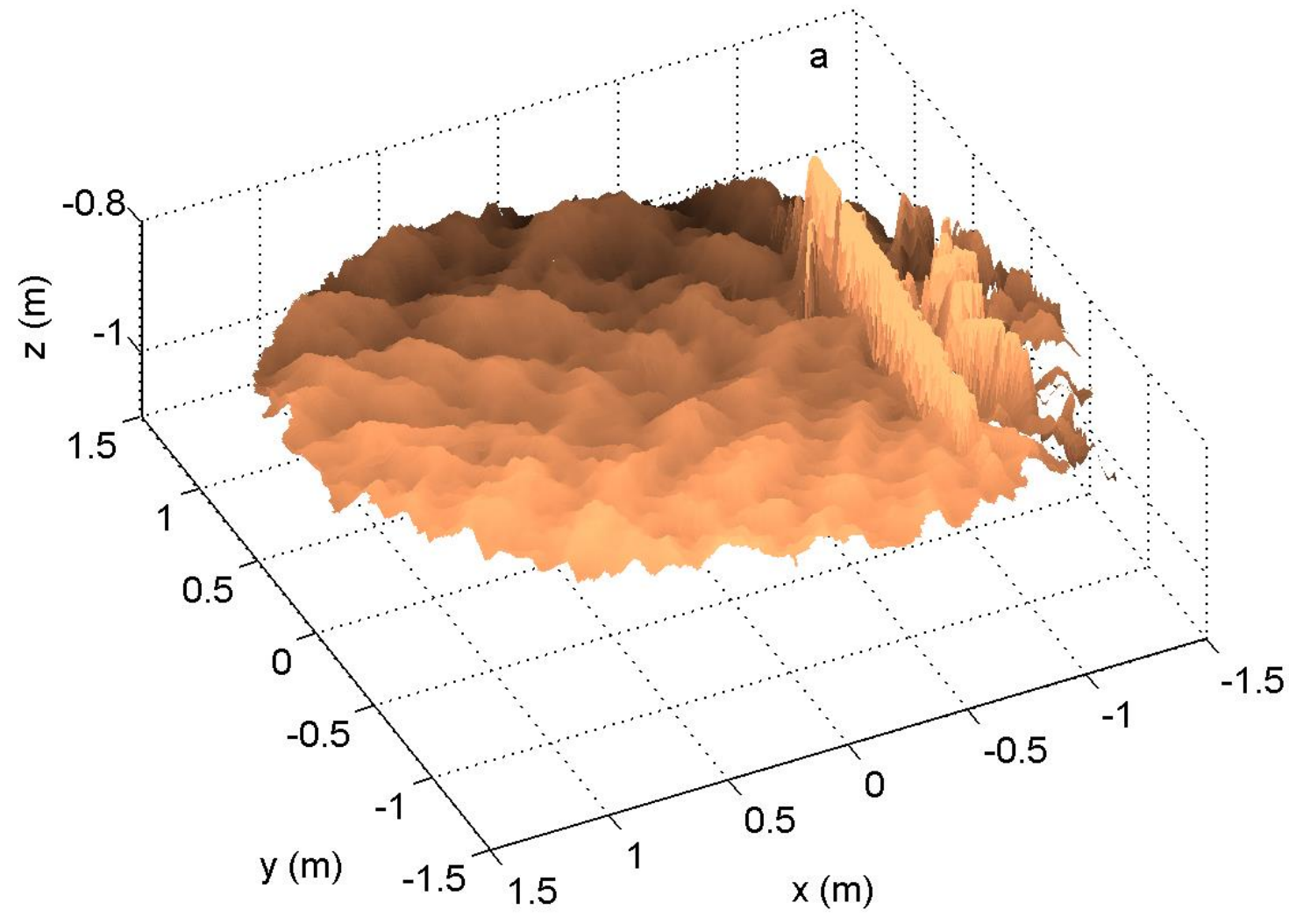

3

4

$5 \quad$ Fig 4a

6 


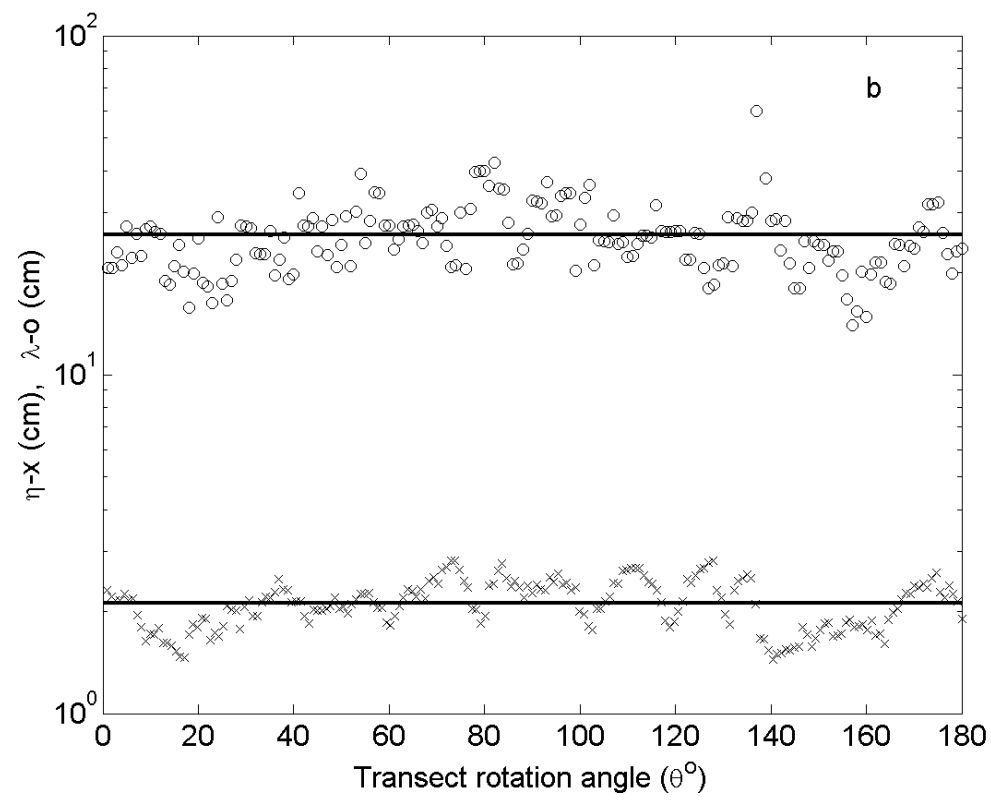

3 Fig 4b

4

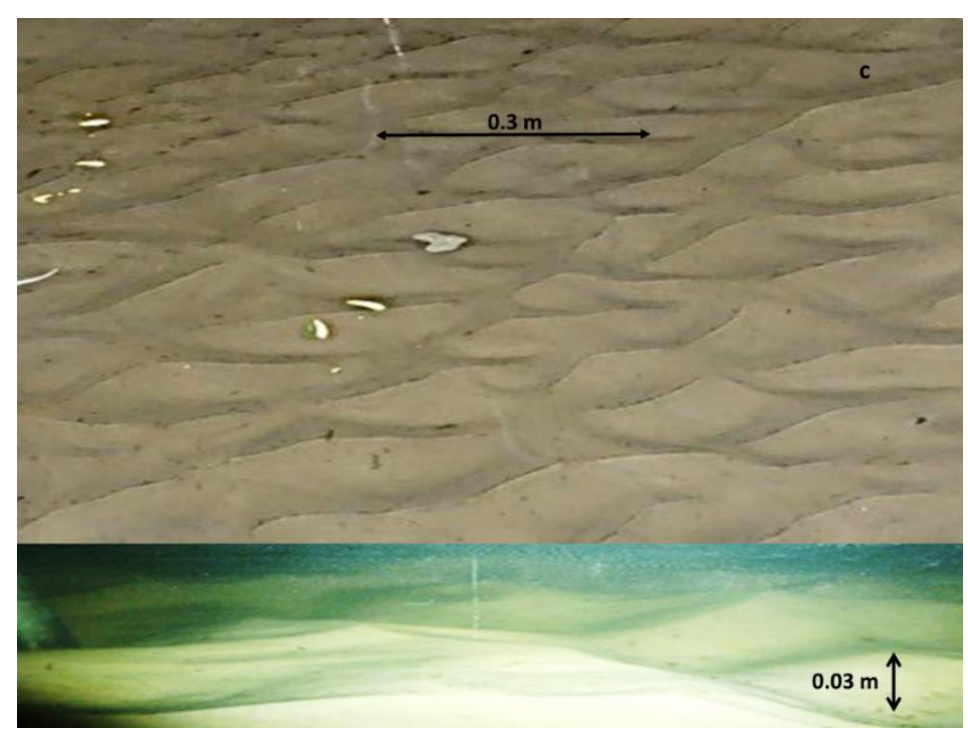

6 Fig 4c

7 

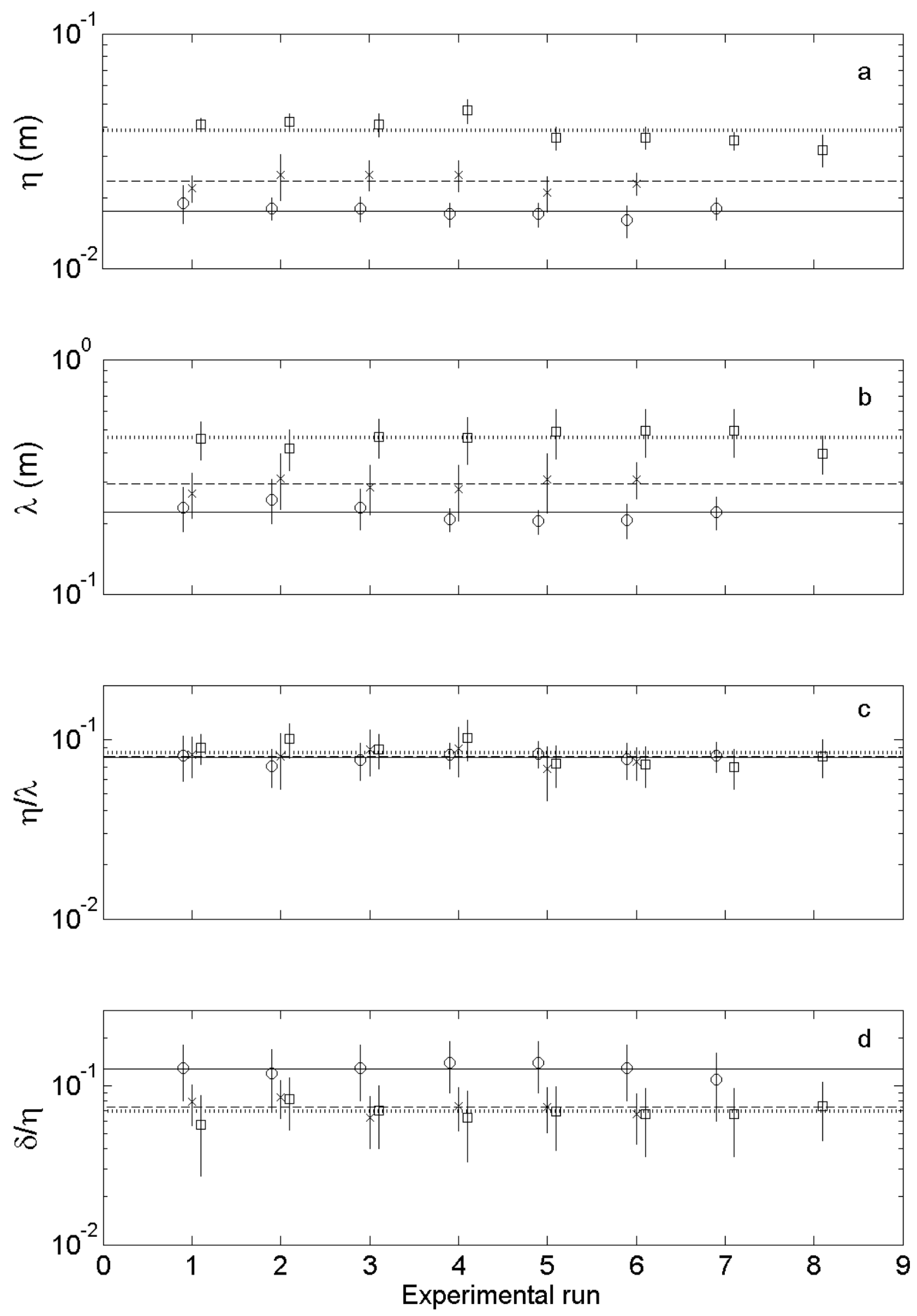

\section{Fig 5}



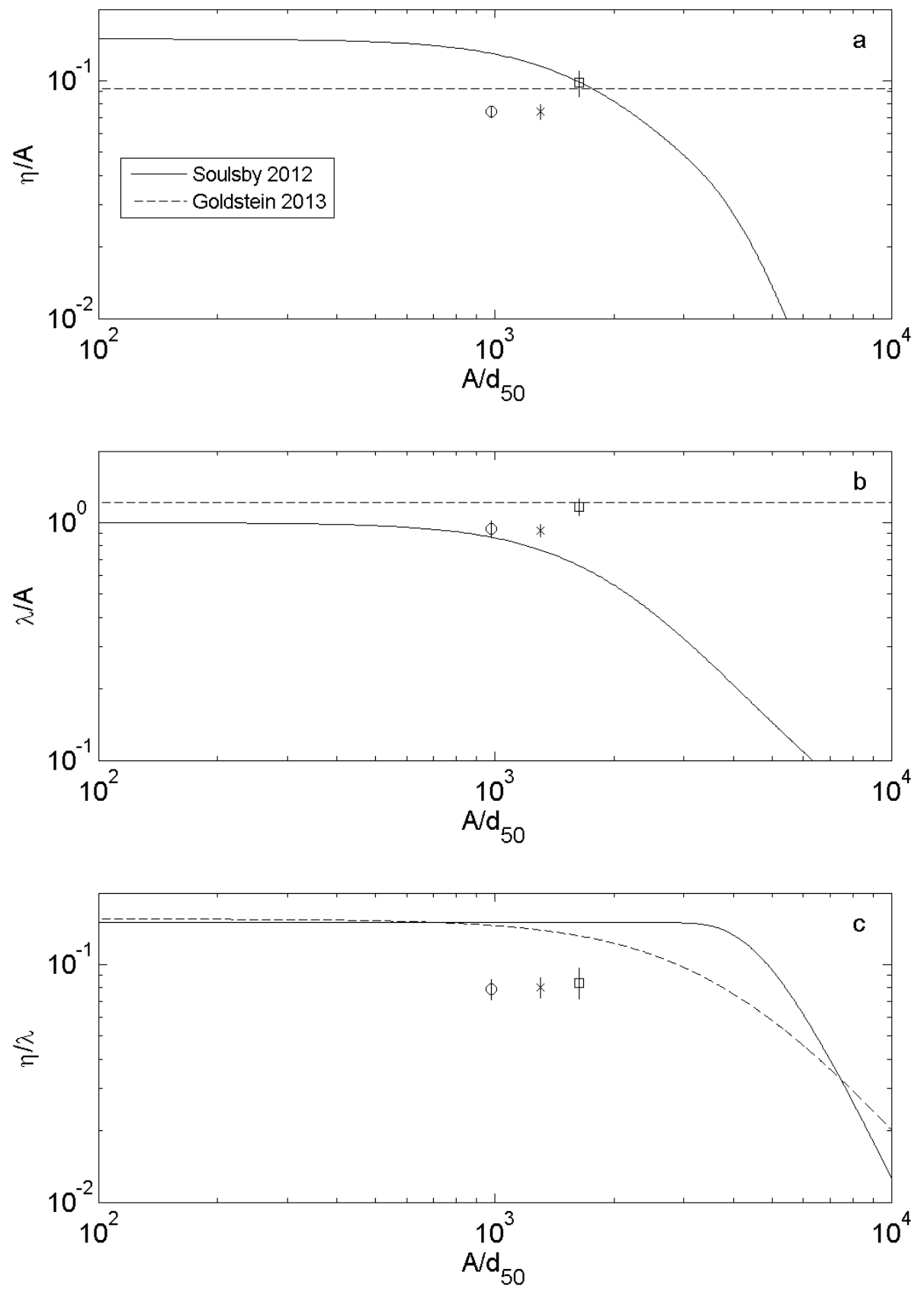

2

Fig 6

3 

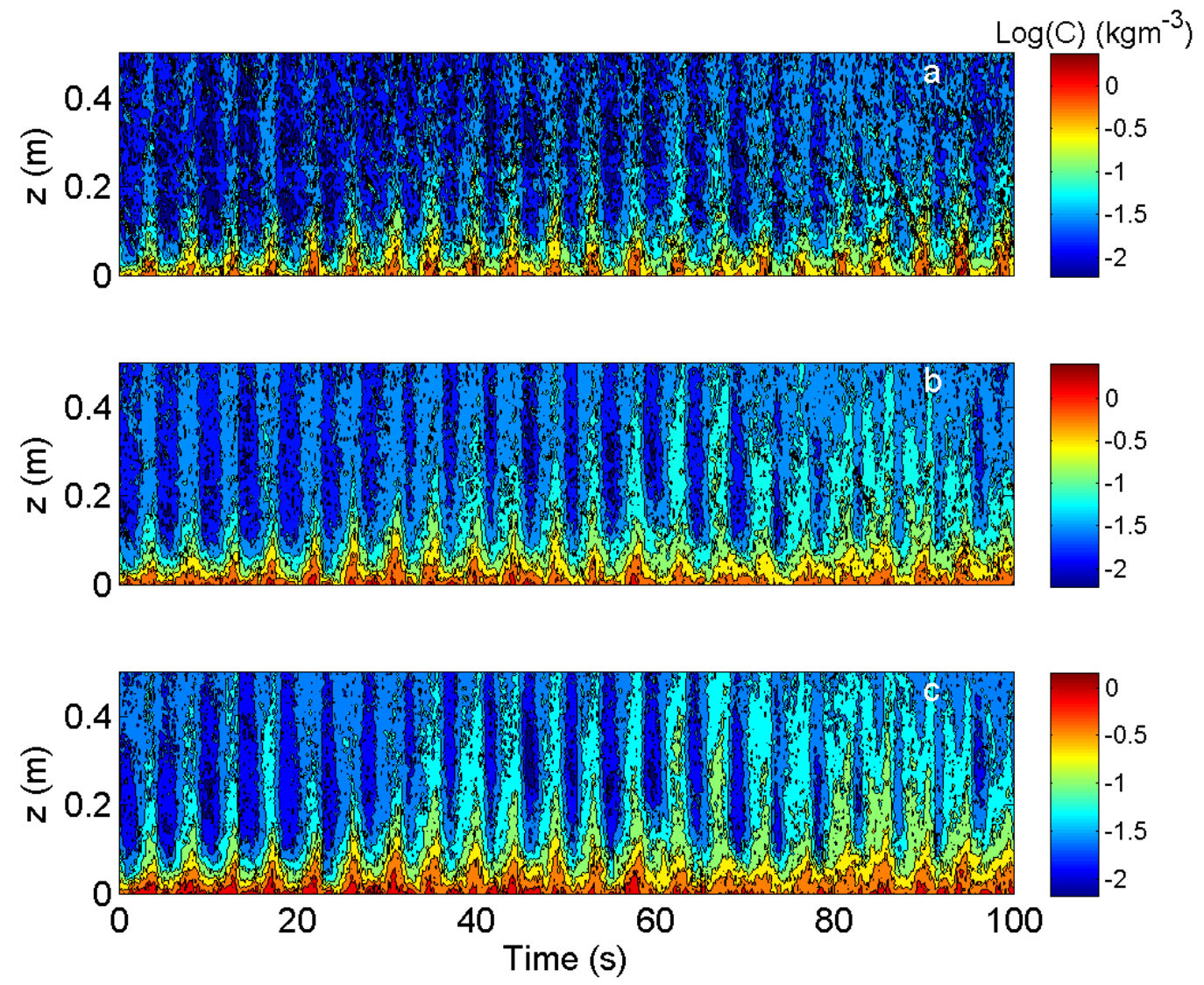

6

$8 \quad$ Fig 7

9 

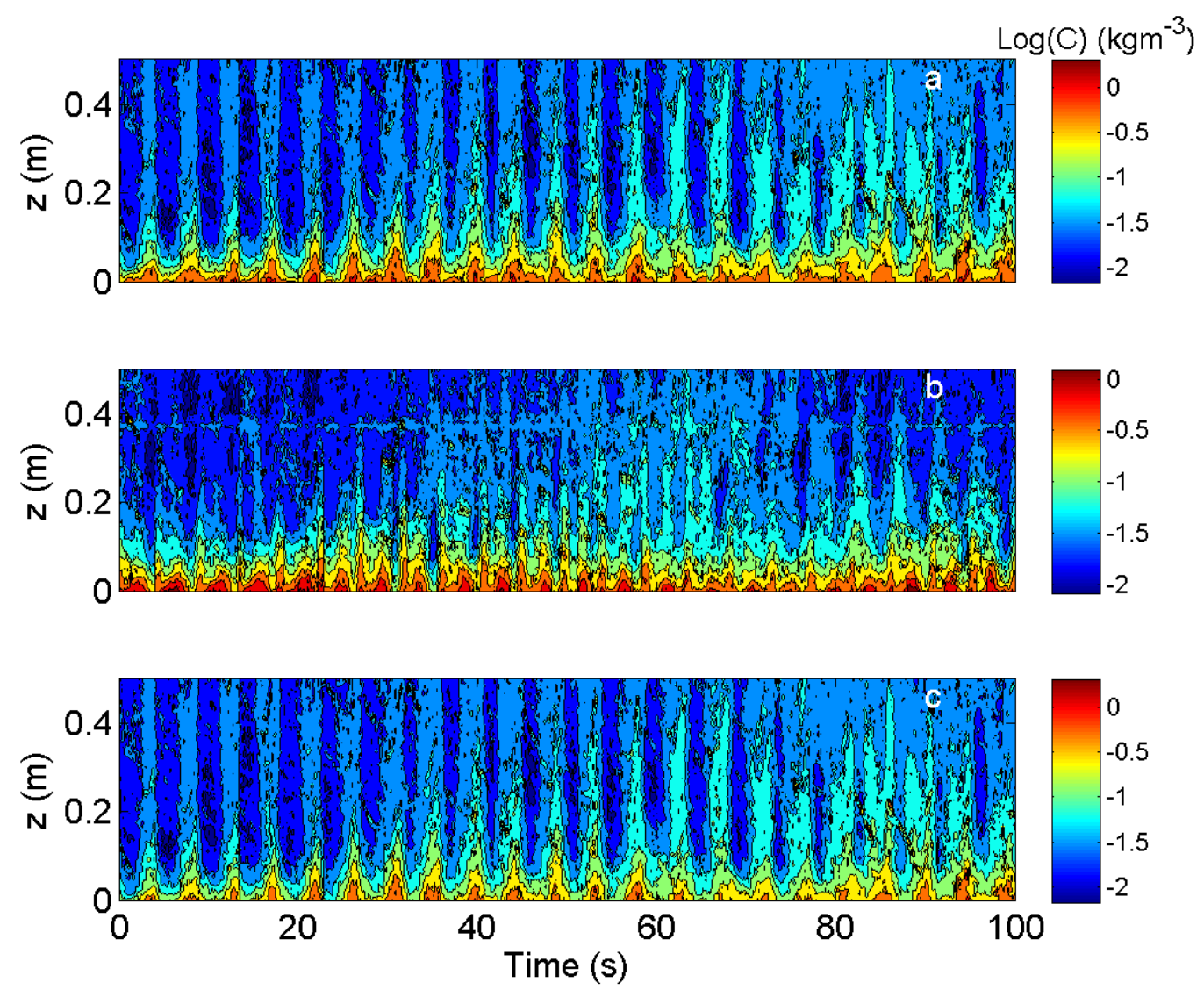

Fig 8 

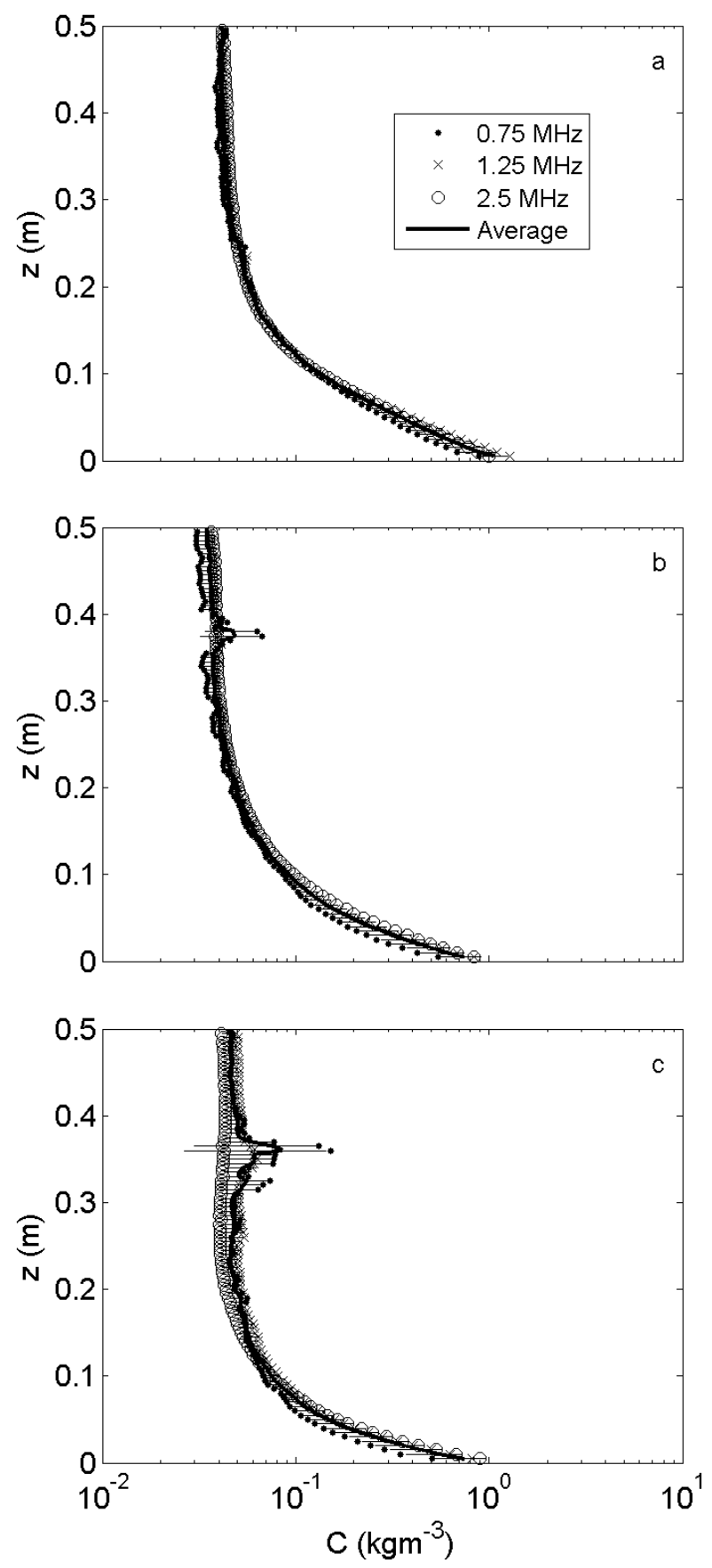

Fig 9 


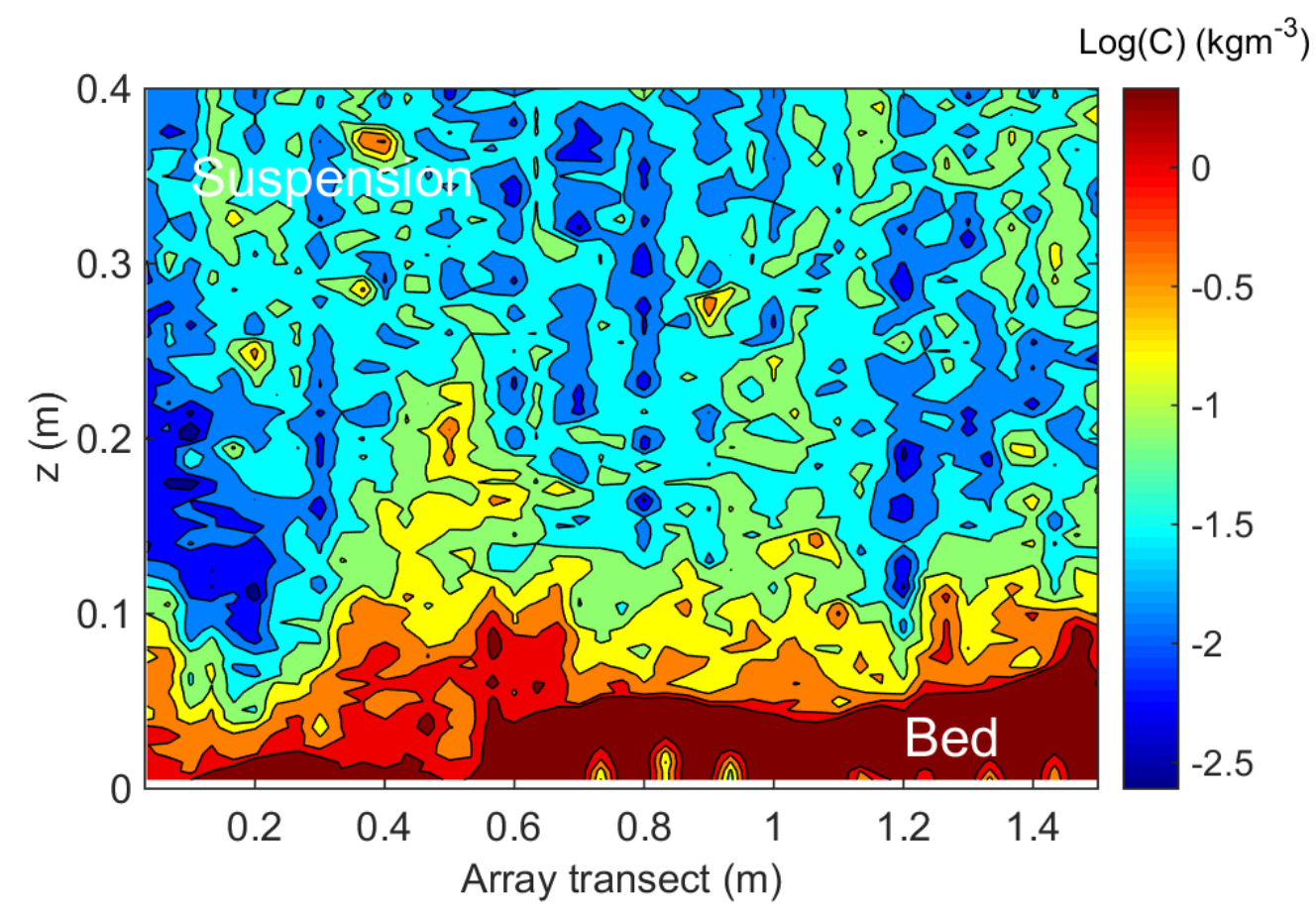

4

$5 \quad$ Fig 10

6 

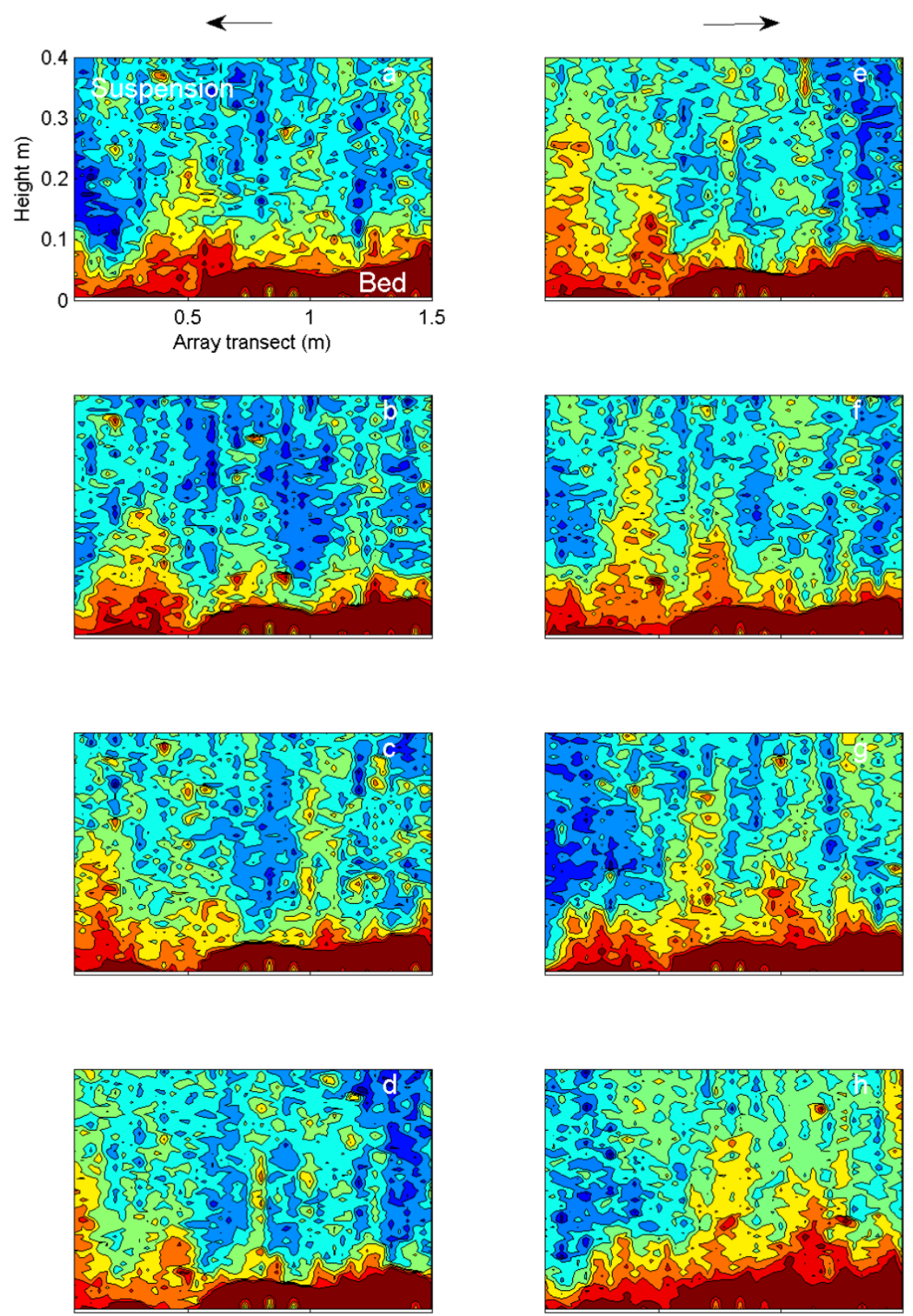

$3 \quad$ Fig 11 

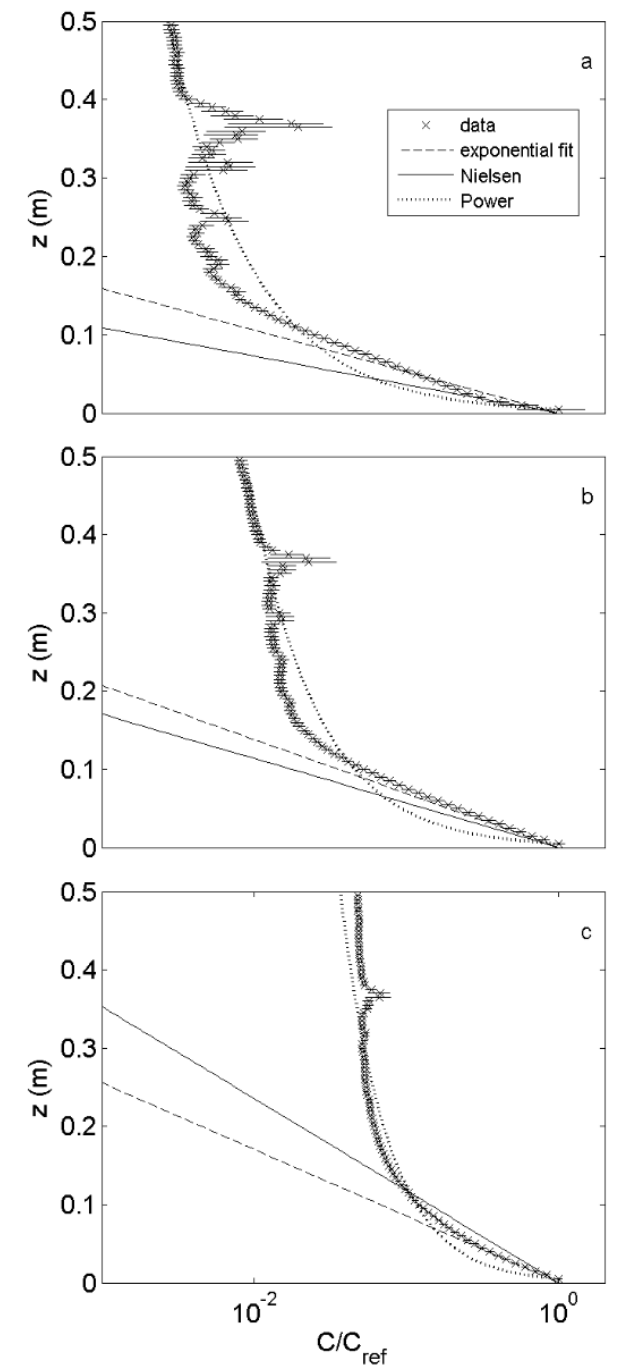

2

$3 \quad$ Fig 12

4 

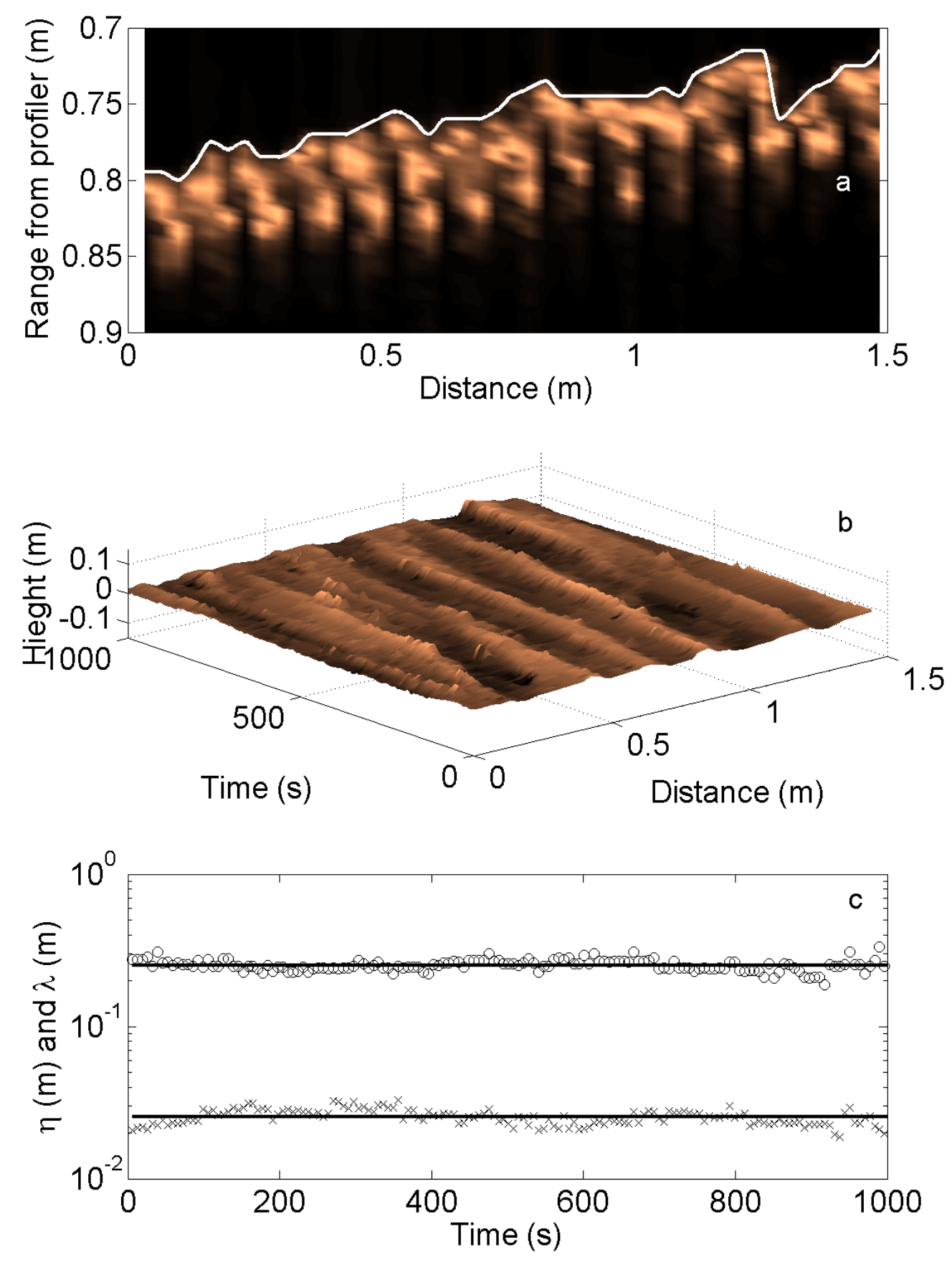

2

$3 \quad$ Fig 13

4 

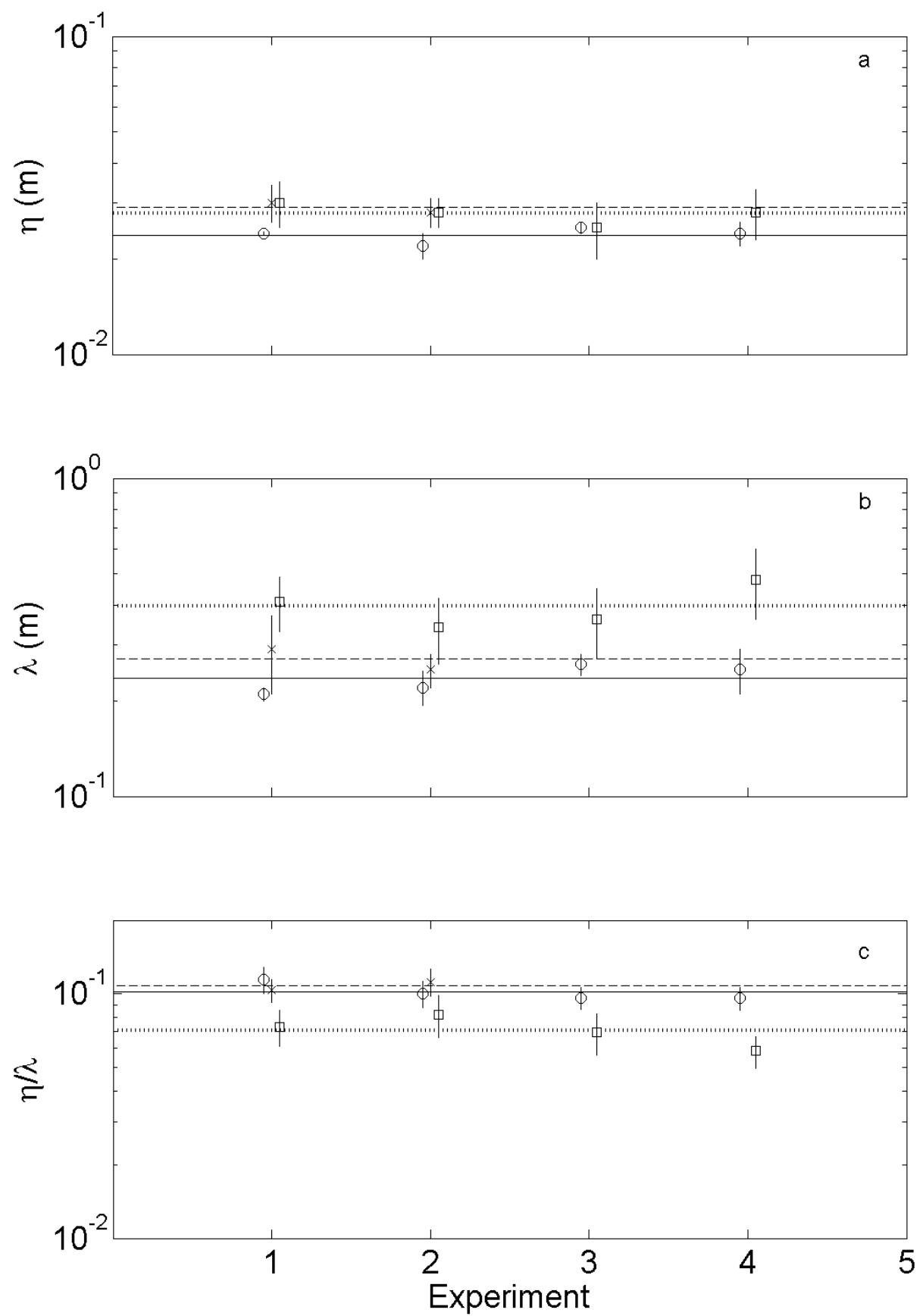

2

$3 \quad$ Fig 14

4 


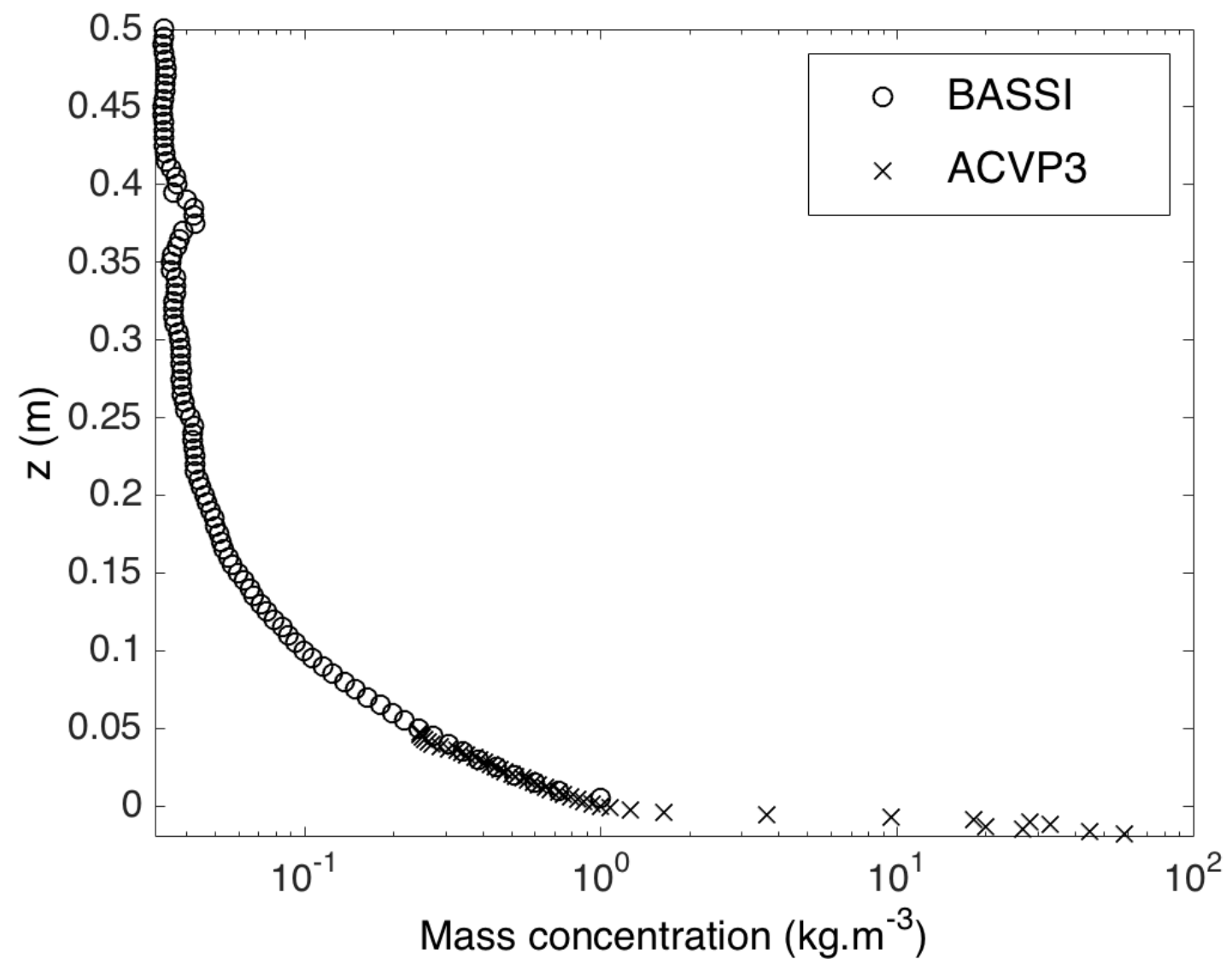

6

$7 \quad$ Fig 15

8 
1

2

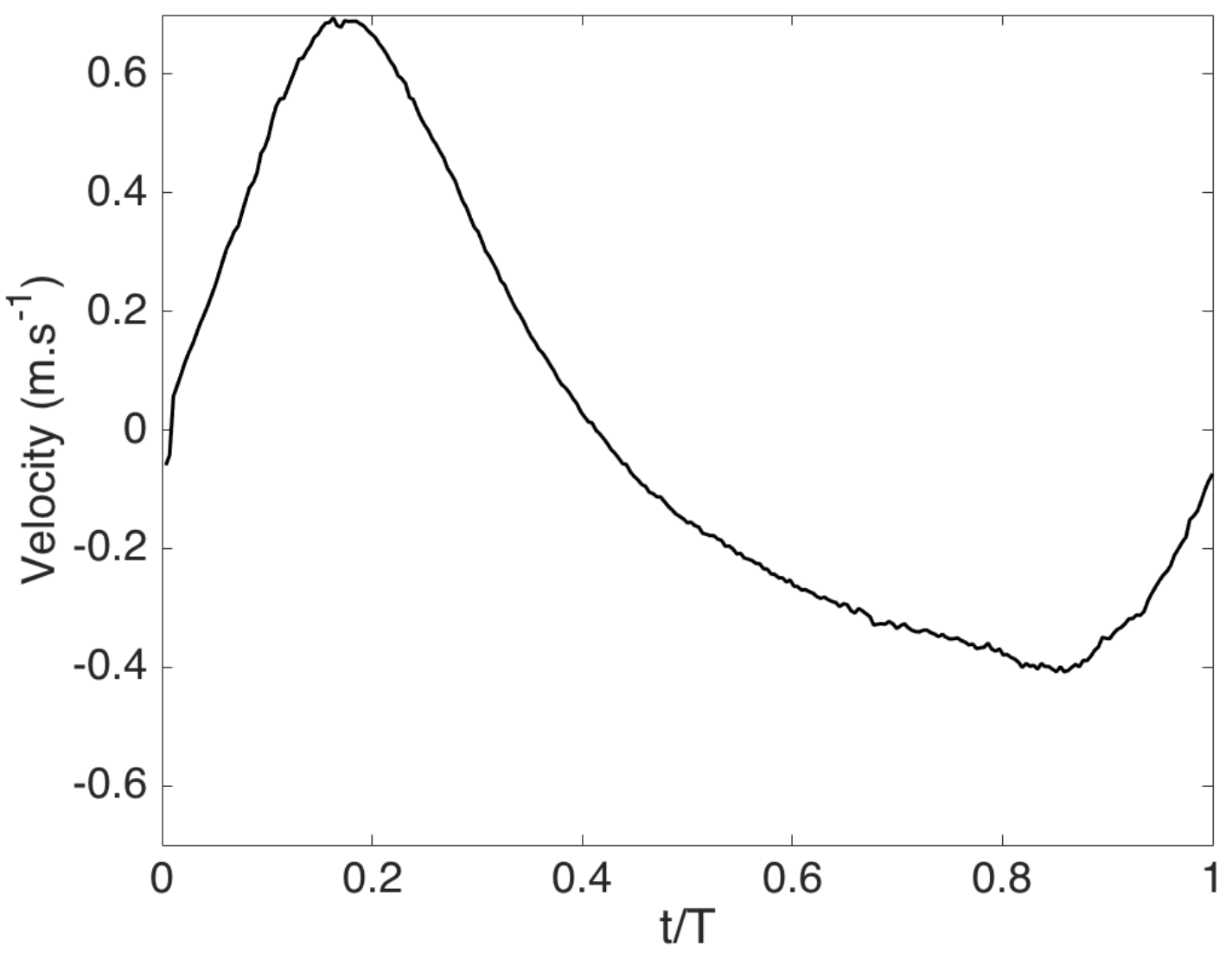

3

Fig 16

5 
6

7

8
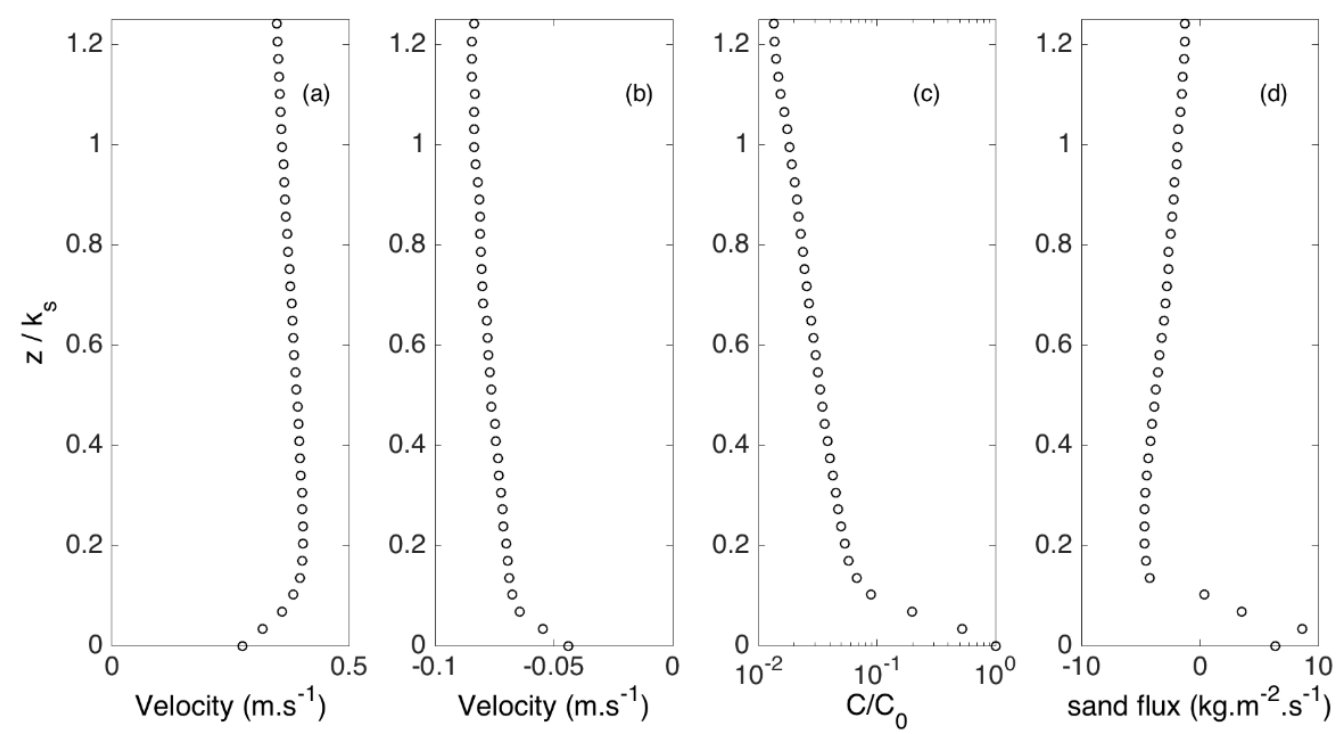

9

10

$11 \quad$ Fig 17

12

13 

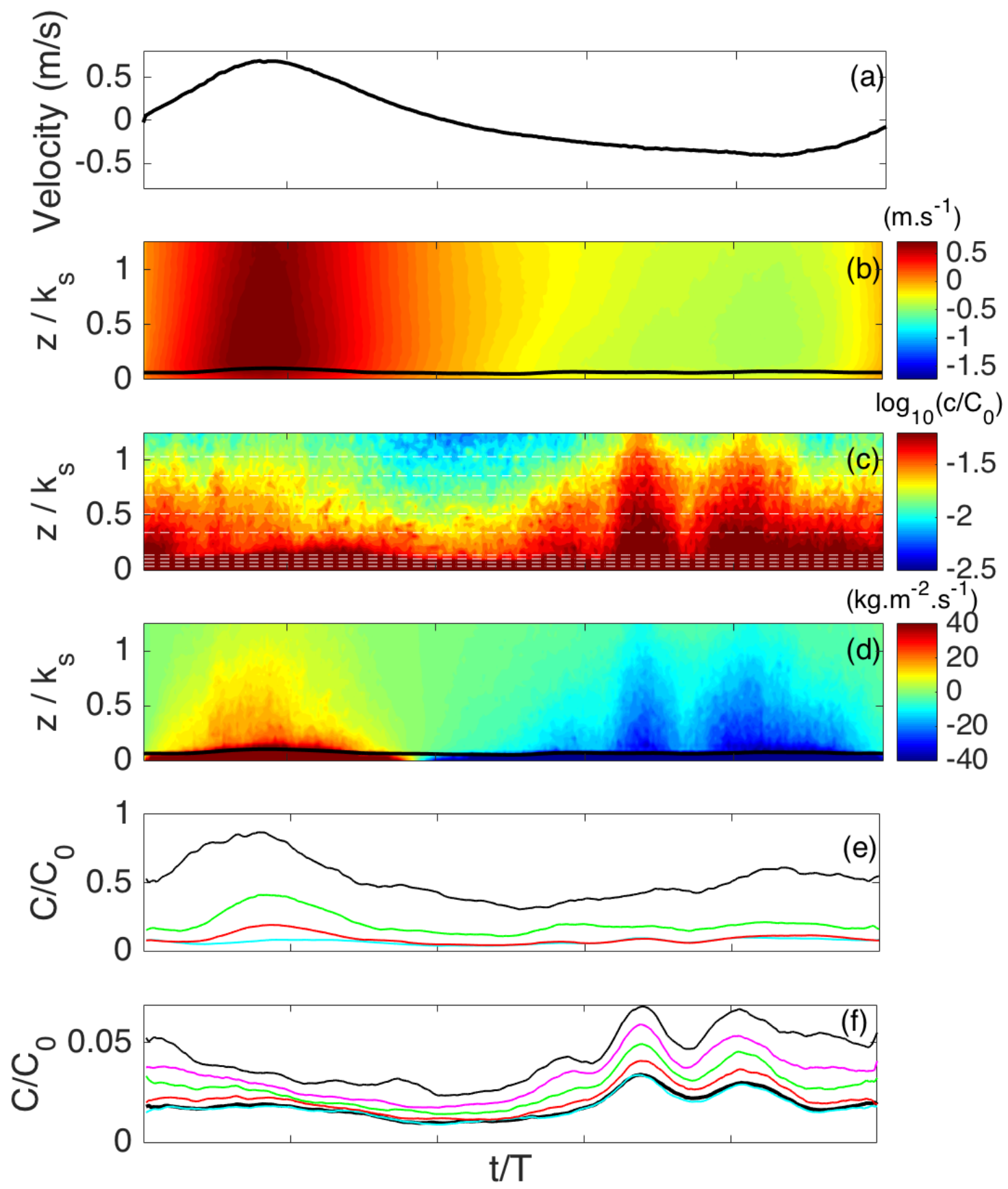

$3 \quad$ Fig 18 

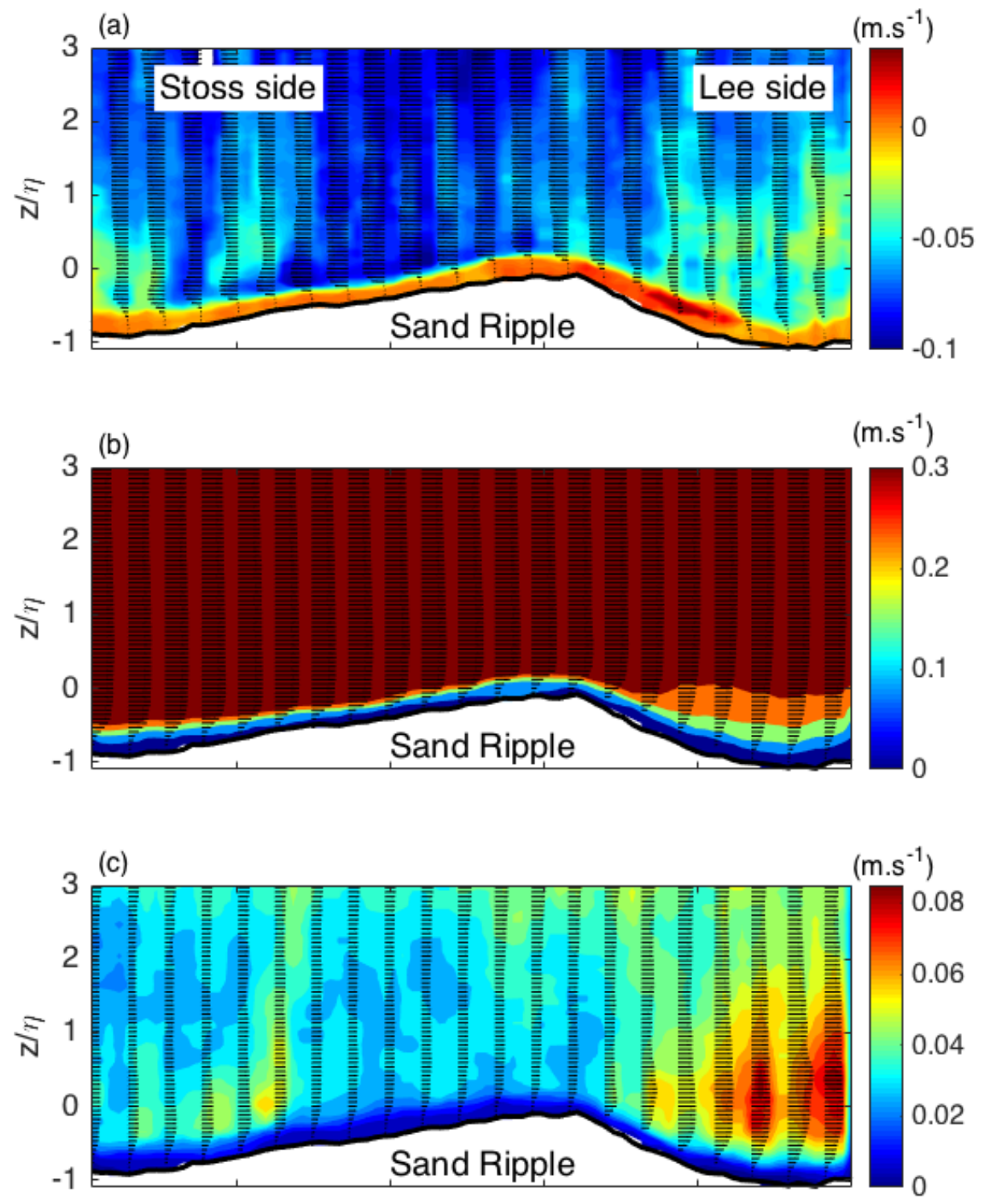

3

4 Fig 19_1 

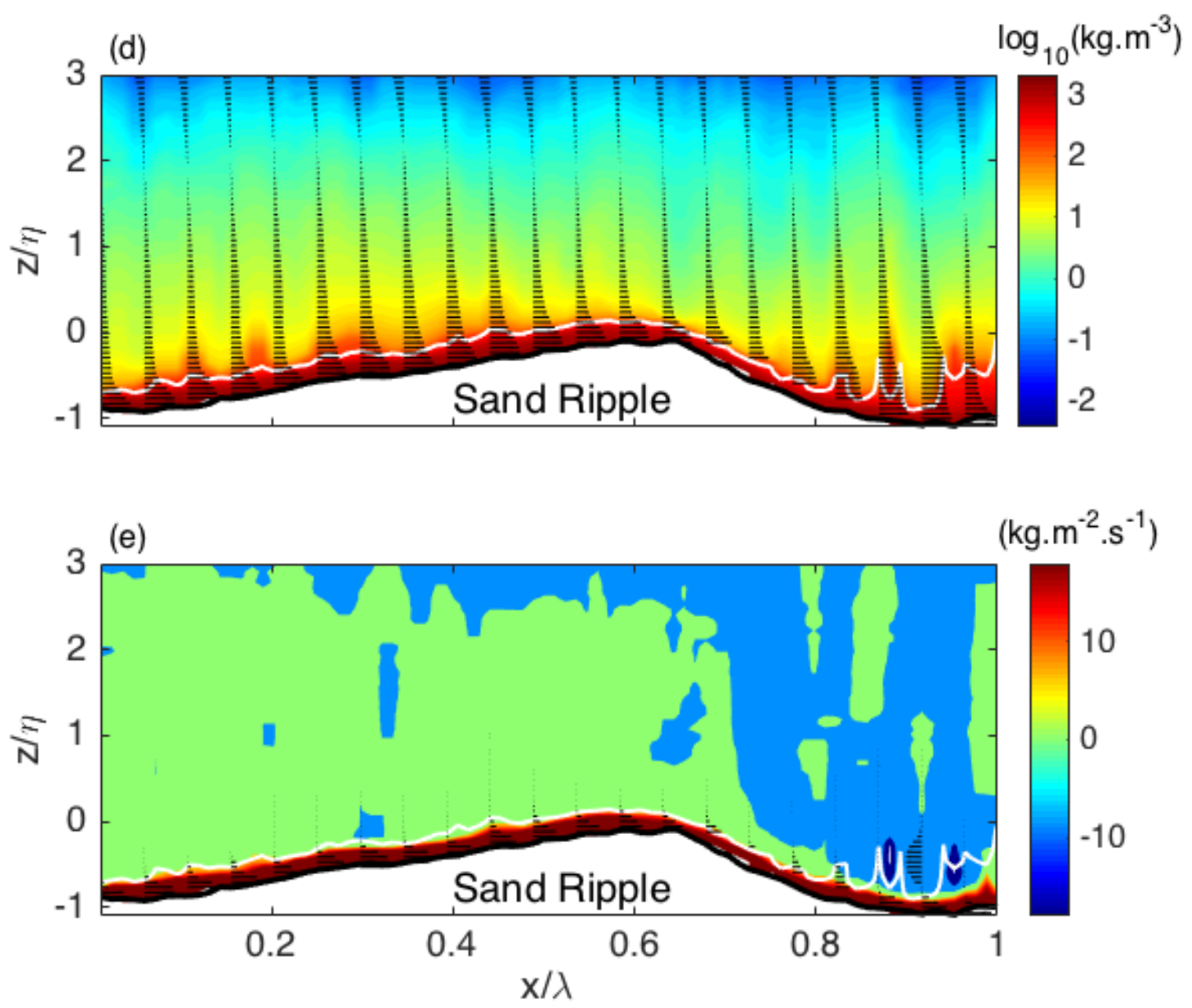

2

3

4 Fig 19_2

5 
2

3

4

5

6
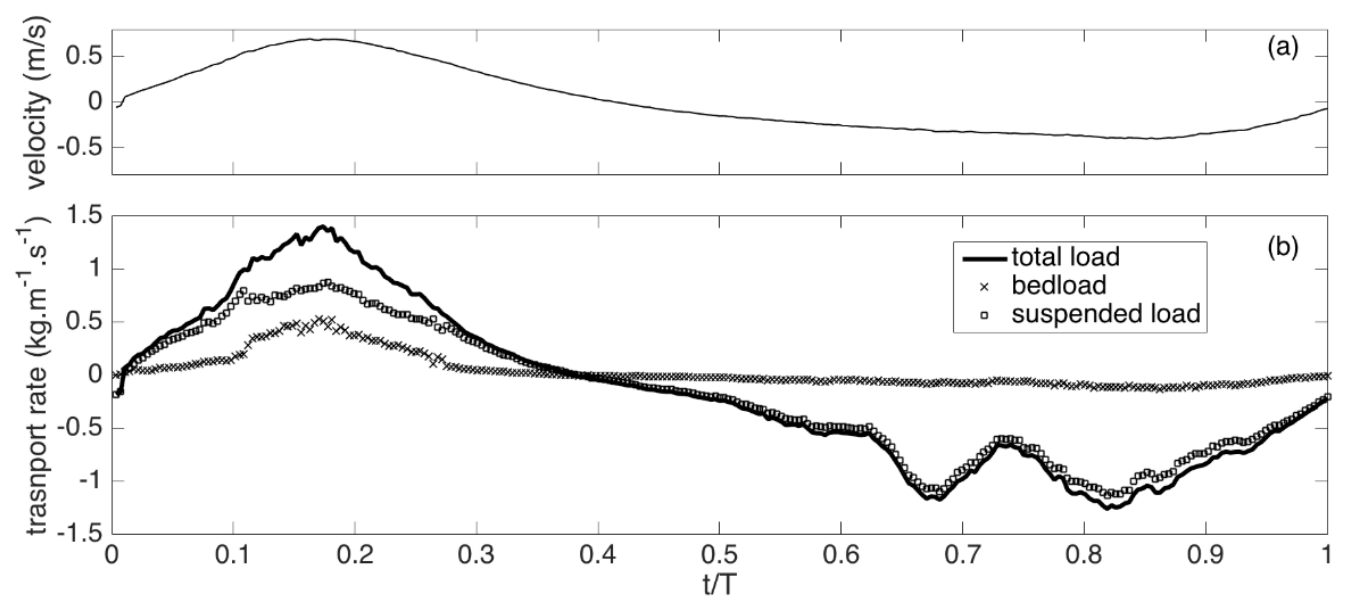

7

8

$9 \quad$ Fig 20

10

11 


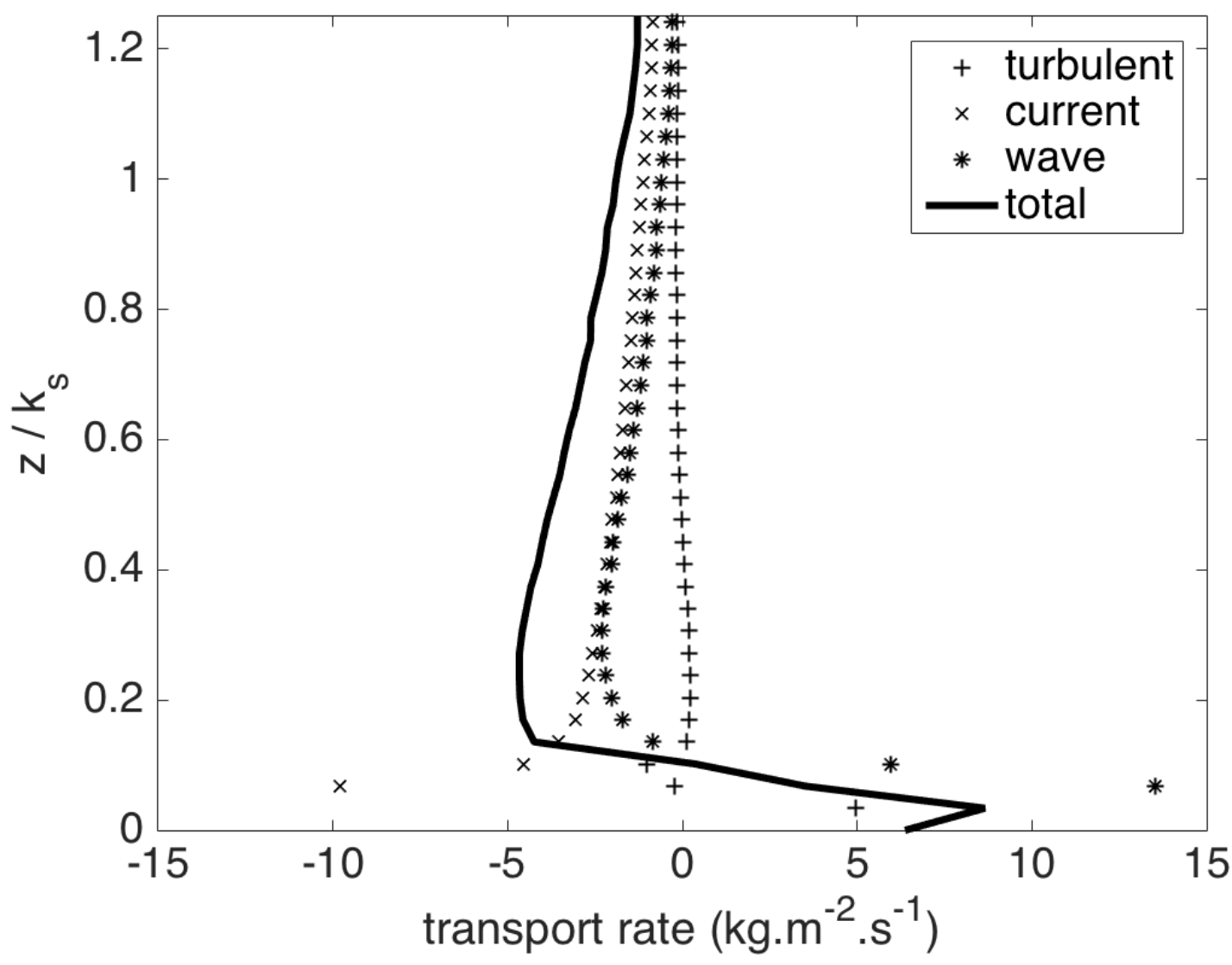

7

$8 \quad$ Fig 21

9 
1

2

3

4

$7 \quad$ Fig 22
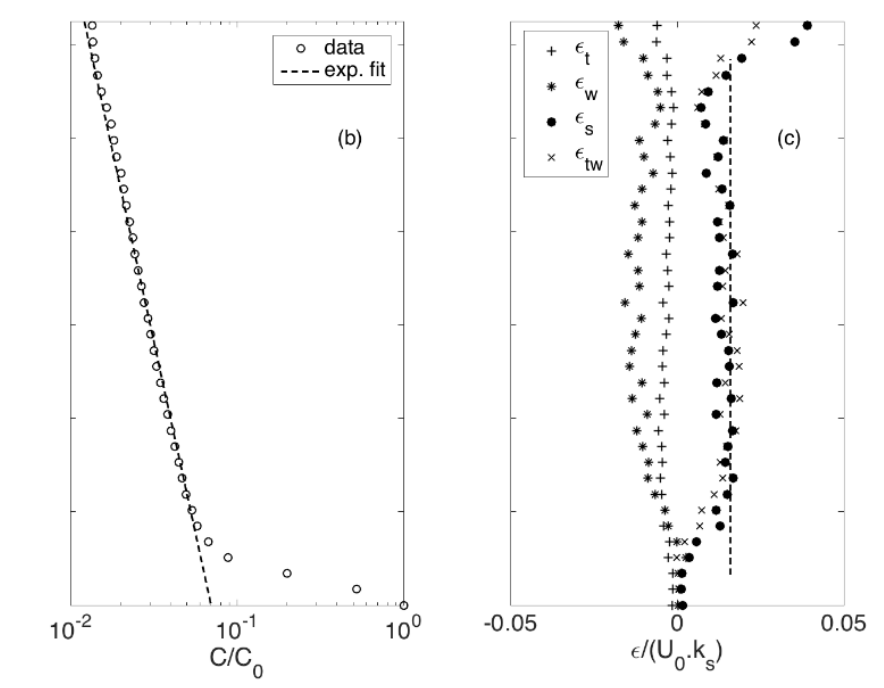

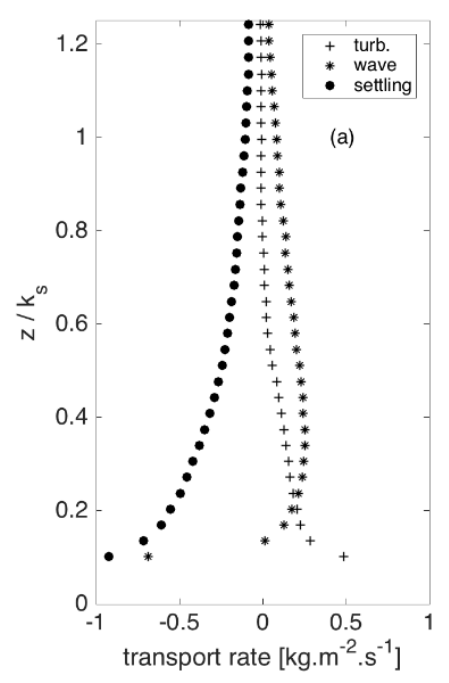

